Homology, Homotopy and Applications, vol.11(1), 2009, pp.141-170

\title{
CLASSIFYING RATIONAL $G$-SPECTRA FOR FINITE $G$
}

\author{
DAVID BARNES \\ (communicated by Brooke Shipley)
}

\begin{abstract}
We give a new proof that for a finite group $G$, the category of rational $G$-equivariant spectra is Quillen equivalent to the product of the model categories of chain complexes of modules over the rational group ring of the Weyl group of $H$ in $G$, as $H$ runs over the conjugacy classes of subgroups of $G$. Furthermore, the Quillen equivalences of our proof are all symmetric monoidal. Thus we can understand categories of algebras or modules over a ring spectrum in terms of the algebraic model.
\end{abstract}

\section{Introduction}

A $G$-equivariant cohomology theory $E^{*}$ is said to be rational if $E^{*}(X)$ is a rational vector space for every $G$-space $X$. For $G$, a finite group, we want to describe the category of rational $G$-equivariant cohomology theories in terms of a simple algebraic model. In particular, we want to understand those cohomology theories with a multiplication and the modules over such a theory. To do so, we give a particular construction of a model category of $G$-spectra whose homotopy category is (equivalent to) the category of rational $G$-equivariant cohomology theories. We show that this model category is symmetric monoidally Quillen equivalent to an explicit algebraic model: the product of the model categories of chain complexes of modules over the rational group ring of the Weyl group of $H$ in $G$, as $H$ runs over the conjugacy classes of subgroups of $G$.

Since our Quillen equivalences are symmetric monoidal, the category of ring spectra is Quillen equivalent to the category of monoids in the algebraic model. Let $W_{G} H$ be the Weyl group of $H$ in $G$, the quotient of the normaliser of $H$ in $G$ by $H$, then a monoid in the category of chain complexes of modules over the rational group ring of $W_{G} H$ is a differential graded $\mathbb{Q}$-algebra with an action (through algebra maps) of the group $W_{G} H$. The category of modules over a ring spectrum will then be Quillen equivalent to modules over a monoid in the algebraic model.

So, if one has a ring spectrum $R$ that one wishes to study, one can look at its image $\tilde{R}$ in the algebraic model and, through the simplicity of the model, perhaps obtain a good description of this monoid. Here one can use the fact that the homology groups of $\tilde{R}$ are isomorphic to the homotopy groups of $R$ to maintain some control of the

Received June 8, 2008, revised September 10, 2008; published on May 8, 2009.

2000 Mathematics Subject Classification: 55N91, 55P42.

Key words and phrases: equivariant cohomology.

This article is available at http://intlpress.com/HHA/v11/n1/a7

Copyright (C) 2009, International Press. Permission to copy for private use granted. 
homology type of $\tilde{R}$. In general, understanding the module category in the algebraic setting will then be a lot simpler than in the topological setting. Conversely, one can take a differential graded algebra with an action of the group $W_{G} H$ and this will correspond, via our Quillen equivalences, to a ring spectrum in the category of $G$-spectra.

We briefly describe the work done previously in this area in order to introduce our result. The category of rational $G$-equivariant cohomology theories is equivalent to the product of the categories of graded modules over the rational group ring of the Weyl group of $H$ in $G$, as $H$ runs over the conjugacy classes of subgroups of $G$; see [GM95, Appendix A] or [May96, Chapter XIX, Section 5].

Let $G \mathcal{M}_{\mathbb{Q}}$ be a model category of $G$-spectra where the weak equivalences are those maps that induce isomorphisms of rational homotopy groups. Then the category of rational $G$-equivariant cohomology theories is (equivalent to) the homotopy category of this model category. The homotopy category of the model category of chain complexes of modules over the rational group ring of $W_{G} H$ is isomorphic to the category of graded modules over the rational group ring of $W_{G} H$. This follows since a rational chain complex is weakly equivalent to its homology, with the latter treated as a chain complex with all differentials zero. Thus, it is natural to ask if the algebraic model (the product of the model categories of chain complexes of modules over the rational group ring of $W_{G} H$, as $H$ runs over the conjugacy classes of subgroups of $G$ ) and $G \mathcal{M}_{\mathbb{Q}}$ are Quillen equivalent. This was proved to be the case in [SS03b, Ex. 5.1.2].

If one starts with a good model category of $G$-spectra, then one can ensure that $G \mathcal{M}_{\mathbb{Q}}$ has a smash product. Similarly, the tensor product of rational chain complexes passes to a monoidal product on the algebraic model. In both cases, the monoidal product induces a product on the homotopy category. So one can ask if these model categories are Quillen equivalent via a series of adjunctions which preserve the monoidal structures on the homotopy categories. That is, is there a series of monoidal Quillen equivalences between $G \mathcal{M}_{\mathbb{Q}}$ and the algebraic model? We construct such a series in this paper and thus we can conclude (using [SS00]) that the various associated categories of algebras and modules in these categories are Quillen equivalent. Our main results are presented in Section 8.

\section{Acknowledgements}

This work consists of material from my Ph.D. thesis, supervised by John Greenlees. I would to thank him for all the help and advice he has given me. I would also like to thank Constanze Roitzheim for a careful reading of an earlier version of this paper and for providing innumerable helpful suggestions.

\section{Outline of the proof}

We begin by explaining [SS03b, Example 5.1.2]: starting from a category of rational $G$-spectra one considers $\mathcal{G}_{\text {top }}=\left\{\Sigma^{\infty} G / H_{+}\right\}$, the set of suspension spectra of the orbit spaces $G / H_{+}$. This provides a set of generators for the homotopy category of rational $G$-spectra. By using the good properties of $L_{\mathbb{Q}} G \mathcal{M}$ we can construct $\mathcal{E}_{t o p}\left(\sigma_{1}, \sigma_{2}\right)$, a symmetric spectrum of functions for each pair, $\left(\sigma_{1}, \sigma_{2}\right)$, of objects of $\mathcal{G}_{\text {top }}$. This collection has a composition rule, $\mathcal{E}_{\text {top }}\left(\sigma_{2}, \sigma_{3}\right) \wedge \mathcal{E}_{\text {top }}\left(\sigma_{1}, \sigma_{2}\right) \rightarrow \mathcal{E}_{\text {top }}\left(\sigma_{1}, \sigma_{3}\right)$. 
Thus we have created an enriched category which we call $\mathcal{E}_{\text {top }}$; it has object set $\mathcal{G}_{\text {top }}$ and the subscript top indicates that this category is of topological origin. We can consider the category of enriched contravariant functors from $\mathcal{E}_{\text {top }}$ to symmetric spectra. We call such a functor a right $\mathcal{E}_{t o p}$-module and these functors and the enriched natural transformations form a category mod- $\mathcal{E}_{t o p}$. If $M$ is one of these enriched functors, then for each pair $\left(\sigma_{1}, \sigma_{2}\right)$ in $\mathcal{G}_{t o p}$ we have symmetric spectra $M\left(\sigma_{1}\right)$ and $M\left(\sigma_{2}\right)$ with an action map $M\left(\sigma_{2}\right) \wedge \mathcal{E}_{\text {top }}\left(\sigma_{1}, \sigma_{2}\right) \rightarrow M\left(\sigma_{1}\right)$. An enriched natural transformation $f: M \rightarrow N$ is then a collection of maps of symmetric spectra $F(\sigma): M(\sigma) \rightarrow N(\sigma)$ compatible with the action maps. If each $f(\sigma)$ is a weak equivalence, we say that $f$ is a weak equivalence of modules and these weak equivalences are part of a model structure on mod- $\mathcal{E}_{t o p}$.

This category of modules is referred to as the collection of 'topological Mackey functors' in [SS03b]. The categories $L_{\mathbb{Q}} G \mathcal{M}$ and mod- $\mathcal{E}_{\text {top }}$ are Quillen equivalent by [SS03b, Theorem 3.3.3]. Since $G$ is finite and we are working rationally, the homotopy groups of $\mathcal{E}_{\text {top }}\left(\sigma_{1}, \sigma_{2}\right)$ (that is, the set of graded maps in the homotopy category of symmetric spectra from $S$ to $\left.\mathcal{E}_{\text {top }}\left(\sigma_{1}, \sigma_{2}\right)\right)$ is concentrated in degree zero, where it takes the value $\underline{A}\left(\sigma_{1}, \sigma_{2}\right)$, a $\mathbb{Q}$-module. Hence $\mathcal{E}_{\text {top }}\left(\sigma_{1}, \sigma_{2}\right)$ is weakly equivalent to an Eilenberg-MacLane spectrum $\mathrm{H} \underline{A}\left(\sigma_{1}, \sigma_{2}\right)$.

From the collection of spectra $\mathrm{H} \underline{A}\left(\sigma_{1}, \sigma_{2}\right)$, we construct a category $\mathrm{H} \underline{A}$, which is enriched over symmetric spectra and one replaces mod- $\mathcal{E}_{\text {top }}$ by the Quillen equivalent category mod-H $\underline{A}$. The collection $\underline{A}\left(\sigma_{1}, \sigma_{2}\right)$ for $\sigma_{1}, \sigma_{2} \in \mathcal{G}_{\text {top }}$ can be thought of as defining a category enriched over $d g \mathbb{Q}$-mod; thus we have a model category mod- $\underline{A}$. There is a zig-zag of Quillen equivalences between mod-H$\underline{A}$ and mod- $\underline{A}$. Thus we have a zig-zag of Quillen equivalences between rational $G$-spectra and an algebraic category.

The algebraic category mod- $\underline{A}$ is actually well-known. Consider the collection of abelian group-enriched functors from $\underline{A}$ to $\mathbb{Q}$-mod. This is better known as the category of rational Mackey functions; hence mod- $\underline{A}$ is the category of differential graded rational Mackey functors. The homotopy category of $\bmod -\underline{A}$ is equivalent to the category of graded rational Mackey functors which, by [GM95, Appendix A], is equivalent to $\prod_{(H) \leqslant G}$ gr. $\mathbb{Q} W_{G} H$-mod. This classification does not consider monoidal structures since the zig-zag between mod-H $\underline{A}$ and mod- $\underline{A}$ passes through a category without a monoidal product. This proof relies upon the fact that the homotopy groups of $\mathcal{E}_{\text {top }}\left(\sigma_{1}, \sigma_{2}\right)$ are concentrated in degree zero.

We now turn to the method of this paper. In the next section we provide a diagram showing the Quillen equivalences that we use. We start by replacing $L_{\mathbb{Q}} G \mathcal{M}$ by the Quillen equivalent category $\prod_{(H) \leqslant G} S_{H}$-mod (where each $S_{H}$ is a commutative ring spectrum) so that we can work one factor at a time. The homotopy category of $S_{H}$-mod is generated by $\sigma_{H}=G / H_{+} \wedge S_{H}$ and for any two $S_{H}$-modules we have a symmetric spectrum function object $\mathcal{E}_{\text {top }}^{H}(X, Y)$. We define $\mathcal{G}_{\text {top }}^{H}$ to be all smash products of $\sigma_{H}$ including $S_{H}$ as the zero-fold smash. Let $\mathcal{E}_{\text {top }}^{H}$ be the symmetric spectrum-enriched category with object set $\mathcal{G}_{\text {top }}^{H}$. The category $S_{H}$-mod is Quillen equivalent to $\mathcal{E}_{\text {top }}^{H}$-mod.

One can now apply an alteration of [Shi07a, Corollary 2.16] (included here as Proposition 6.1) to construct a category $\mathcal{E}_{t}^{H}$ from $\mathcal{E}_{\text {top }}^{H}$. This new category will be enriched over rational chain complexes with its set of objects given by $\mathcal{G}_{\text {top }}^{H}$. The 
$t$ indicates that we have come from the topological side but are now working in an algebraic setting. We can consider enriched functors from $\mathcal{E}_{t}^{H}$ to rational chain complexes; this category will be denoted $\bmod -\mathcal{E}_{t}^{H}$.

Now we begin our work from the other end. $\mathrm{Ch}\left(\mathbb{Q} W_{G} H\right.$-mod) is generated by the object $\mathbb{Q} W_{G} H$, so we let $\mathcal{G}_{a}^{H}$ be the set of all tensor products of $\mathbb{Q} W_{G} H$ (with $\mathbb{Q}$ as the zero-fold product). Analogously to the topological setting, the set $\mathcal{G}_{a}^{H}$ is the object set for a category $\mathcal{E}_{a}^{H}$, which is enriched over rational chain complexes. We can then replace our algebraic model by the Quillen equivalent category mod- $\mathcal{E}_{a}^{H}$. The $a$ indicates we have come from the algebraic model.

So far this process has been formal, now we use some specific information about $\mathcal{E}_{t}^{H}$ and $\mathcal{E}_{a}^{H}$ to achieve a comparison between them. The comparison we will use is the notion of a quasi-isomorphism of categories enriched over rational chain complexes. Given two such categories $\mathcal{C}$ and $\mathcal{D}$, an enriched functor $F: \mathcal{C} \rightarrow \mathcal{D}$ is a quasi-isomorphism if $F$ induces an isomorphism on the classes of objects and each $F\left(\sigma_{1}, \sigma_{2}\right): \mathcal{C}\left(\sigma_{1}, \sigma_{2}\right) \rightarrow \mathcal{D}\left(F \sigma_{1}, F \sigma_{2}\right)$, is a homology isomorphism. $\left(F\left(\sigma_{1}, \sigma_{2}\right)\right.$ is a map in the category of rational chain complexes.) A quasi-isomorphism induces a Quillen equivalence between mod-C and $\bmod -\mathcal{D}$.

In our case we define an isomorphism $F$ on the object sets by sending the $i$-fold product of $\mathbb{Q} W_{G} H$ to the $i$-fold product of $G / H \wedge S_{H}$. We then prove that the homology of $\mathcal{E}_{t}^{H}\left(F \sigma_{1}, F \sigma_{2}\right)$ is concentrated in degree zero and is isomorphic to $\mathcal{E}_{a}^{H}\left(\sigma_{1}, \sigma_{2}\right)$. Since we are enriched over rational chain complexes, it follows that $\mathcal{E}_{t}^{H}$ and $\mathcal{E}_{a}^{H}$ are quasi-isomorphic and hence that there is a zig-zag of Quillen equivalences between $\bmod -\mathcal{E}_{t}^{H}$ and $\bmod -\mathcal{E}_{a}^{H}$. Thus the category of rational $G$-spectra is Quillen equivalent to $\prod_{(H) \leqslant G} \mathrm{Ch}\left(\mathbb{Q} W_{G} H\right.$-mod). If we can prove that the quasi-isomorphisms between $\mathcal{E}_{t}$ and $\mathcal{E}_{a}$ respect the monoidal structure, then it will follow that the zigzag between rational $G$-spectra and the algebraic model consists of monoidal Quillen equivalences.

The difference between our method and that of $[\mathbf{S S 0 3} \mathbf{b}]$ is the ordering of the work. We split the category, move to Mackey functors (modules over a symmetric spectrum-enriched category), translate to algebra and then use the fact that $\mathcal{E}_{t}^{H}$ has homology concentrated in degree zero, whereas [SS03b] goes to Mackey functors first, uses the fact that $\mathcal{E}_{\text {top }}$ has homotopy concentrated in degree zero, moves to algebra and then splits the category. The category $\mathcal{E}_{t}^{H}$ is constructed so that its homology groups are isomorphic to the homotopy groups of $\mathcal{E}_{\text {top }}^{H}$; hence the two methods use the same information, just in different contexts.

The result could have been proved without using the splitting theorem. The advantage to using this extra step is that $S_{H}$-mod is generated by a single object, which makes $\mathcal{E}_{\text {top }}^{H}$ and $\mathcal{E}_{t}^{H}$ easier to work with.

\section{Organisation}

We describe the results of each section and display the Quillen equivalences that we use. Left adjoints will be placed on top, all adjunctions are symmetric (weak) monoidal and furthermore all left adjoints except $L^{\prime}$ are strong symmetric monoidal functors. 
In Section 4, we examine our algebraic category and describe the Quillen equivalence between $\operatorname{Ch}\left(\mathbb{Q} W_{G} H\right.$-mod $)$ and $\bmod \mathcal{E}_{a}^{H}$, a category of modules over an enriched category

$$
(-) \otimes_{\mathcal{E}_{a}^{H}} \mathcal{G}_{a}^{H}: \bmod -\mathcal{E}_{a}^{H} \rightleftarrows \mathrm{Ch}\left(\mathbb{Q} W_{G} H \text {-mod }\right): \underline{\operatorname{Hom}}\left(\mathcal{G}_{a}^{H},-\right) .
$$

We then develop a category of rational spectra in Section 5 and 'split' this category into a product $\prod_{(H) \leqslant G} S_{H}$-mod so that it looks more like the algebraic category. We end this section by replacing $S_{H}$-mod by mod- $\mathcal{E}_{t o p}^{H}$, modules over a category enriched in symmetric spectra. This step requires a little work.

$$
G \mathcal{M}_{\mathbb{Q}} \stackrel{\Delta}{\rightleftarrows} \prod_{(H) \leqslant G} L_{E\langle H\rangle} G \mathcal{M}_{\mathbb{Q}} \stackrel{S_{H} \wedge(-)}{\rightleftarrows} \prod_{(H) \leqslant G} S_{H}-\bmod \underset{\underline{(-) \wedge_{\mathcal{E}_{t o p}^{H}} \mathcal{G}_{t o p}^{H}}}{\stackrel{\text { Hom }\left(\mathcal{G}_{t o p}^{H},-\right)}{\rightleftarrows}} \prod_{(H) \leqslant G} \bmod -\mathcal{E}_{t o p}^{H}
$$

Now we give the functors from Section 6 (though we omit the identity adjunction of Lemma 6.2). The functors below are adaptations of those in [Shi07a] and we define $\mathcal{E}_{t}^{H}$ to be $D \phi^{*} N \tilde{\mathbb{Q}} \mathcal{E}_{\text {top }}^{H}$, a category enriched over rational chain complexes.

$$
\bmod -\mathcal{E}_{t o p}^{H} \underset{U^{\prime}}{\stackrel{\tilde{\mathbb{Q}}}{\rightleftarrows}} \bmod -\tilde{\mathbb{Q}} \mathcal{E}_{t o p}^{H} \underset{\phi^{*} N}{\stackrel{L^{\prime}}{\leftrightarrows}} \bmod -\phi^{*} N \tilde{\mathbb{Q}} \mathcal{E}_{t o p}^{H} \underset{R^{\prime}}{\stackrel{D}{\rightleftarrows}} \bmod -D \phi^{*} N \tilde{\mathbb{Q}} \mathcal{E}_{t o p}^{H}
$$

In Section 7 we prove that the homology category of the enriched category $\mathcal{E}_{t}^{H}$ is isomorphic to the category $\mathcal{E}_{a}^{H}$. Let $\psi: \mathcal{E}_{a}^{H} \rightarrow \mathrm{H}_{*} \mathcal{E}_{t}^{H}$ be this isomorphism (Theorem 7.4) and write $\left(\psi^{-1}\right)^{*}$ for the left adjoint to $\psi^{*}$. Let $i: C_{0} \mathcal{E}_{t}^{H} \rightarrow \mathcal{E}_{t}^{H}$ and $p: C_{0} \mathcal{E}_{t}^{H} \rightarrow \mathrm{H}_{*} \mathcal{E}_{t}^{H}$ be the maps constructed in Corollary 7.6. Then we have the Quillen equivalences below.

$$
\bmod -\mathcal{E}_{t}^{H} \stackrel{(-) \otimes_{C_{0} \mathcal{E}_{t}^{H}} \mathcal{E}_{t}^{H}}{\leftrightarrows} \bmod -C_{0} \mathcal{E}_{t}^{H}{ }^{(-) \otimes_{C_{0}} \mathcal{E}_{t}^{H} \mathrm{H}_{*} \mathcal{E}_{t}^{H}} \underset{p^{*}}{\rightleftarrows} \bmod -\mathrm{H}_{*} \mathcal{E}_{t}^{H} \stackrel{\left(\psi^{-1}\right)^{*}}{\leftrightarrows} \bmod -\mathcal{E}_{a}^{H}
$$

We put these results together to obtain the main result and consider the categories of algebras and modules in Section 8.

In the table below, we provide a list of the enriched categories that we use in this paper. The object set $\mathcal{G}_{a}^{H}$ is the set of all tensor products of $\mathbb{Q} W_{G} H$ and $\mathcal{G}_{\text {top }}^{H}$ is the set of all smash products of $\left(\widehat{c} G / H_{+}\right) \wedge S_{H}$ (in the category of $S_{H^{-}}$-modules). For $S_{H^{-}}$ modules $X$ and $Y, \underline{\operatorname{Hom}}(X, Y)=\operatorname{Sing} \mathbb{U}\left(i^{*} \mathbb{N}^{\#} F_{S_{H}}(X, Y)\right)^{G}$ as we will explain later.

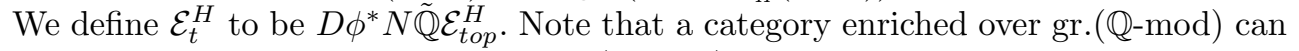
also be considered as enriched over $\mathrm{Ch}(\mathbb{Q}$-mod).

\section{The algebraic category}

We study the algebraic model in detail and the main result of this section is Theorem 4.9. In a very rough sense, this results puts the information of the algebraic model into a standard form, suitable for later comparisons. We also use this section as an introduction to the methods of this paper. 


\begin{tabular}{|c|c|c|c|}
\hline Name & Enrichment & Object Set & Morphism Object \\
\hline $\mathcal{E}_{a}^{H}$ & $\mathrm{Ch}(\mathbb{Q}-$ mod $)$ & $\mathcal{G}_{a}^{H}$ & $\operatorname{Hom}_{\mathbb{Q}}(a, b)^{W_{G} H}$ \\
\hline $\mathcal{E}_{t o p}^{H}$ & $S p^{\Sigma}$ & $\mathcal{G}_{\text {top }}^{H}$ & $\underline{\operatorname{Hom}}(X, Y)$ \\
\hline$\tilde{\mathbb{Q}} \mathcal{E}_{t o p}^{H}$ & $S p^{\Sigma}(\mathrm{s} \mathbb{Q}-\mathrm{mod})$ & $\mathcal{G}_{\text {top }}^{H}$ & $\tilde{\mathbb{Q}} \underline{\operatorname{Hom}}(X, Y)$ \\
\hline$\phi^{*} N \tilde{\mathbb{Q}} \mathcal{E}_{t o p}^{H}$ & $S p^{\Sigma}\left(\mathrm{Ch}(\mathbb{Q}-\text { mod })_{+}\right)$ & $\mathcal{G}_{\text {top }}^{H}$ & $\phi^{*} N \tilde{\mathbb{Q}} \underline{\operatorname{Hom}}(X, Y)$ \\
\hline $\mathcal{E}_{t}^{H}$ & $\mathrm{Ch}(\mathbb{Q}-$ mod $)$ & $\mathcal{G}_{\text {top }}^{H}$ & $D \phi^{*} N \tilde{\mathbb{Q}} \underline{\operatorname{Hom}}(X, Y)$ \\
\hline$\pi_{*} \mathcal{E}_{t o p}^{H}$ & $\operatorname{gr} .(\mathbb{Q}-$ mod $)$ & $\mathcal{G}_{\text {top }}^{H}$ & $\pi_{*} \underline{\operatorname{Hom}}(X, Y)$ \\
\hline Ho $\mathcal{E}_{\text {top }}^{H}$ & gr. $(\mathbb{Q}-$ mod $)$ & $\mathcal{G}_{\text {top }}^{H}$ & {$[X, Y]_{*}^{S_{H}}$} \\
\hline$\pi_{*} \mathcal{G}_{\text {top }}^{H}$ & gr. $(\mathbb{Q}-$ mod $)$ & $\mathcal{G}_{\text {top }}^{H}$ & $\operatorname{Hom}_{\mathbb{Q}}\left(\pi_{*}^{H}(X), \pi_{*}^{H}(Y)\right)^{W_{G} H}$ \\
\hline$C_{0} \mathcal{E}_{t}^{H}$ & $\operatorname{Ch}(\mathbb{Q}-$ mod $)$ & $\mathcal{G}_{\text {top }}^{H}$ & $C_{0} \mathcal{E}_{t}^{H}(X, Y)$ \\
\hline $\mathrm{H}_{*} \mathcal{E}_{t}^{H}$ & $\operatorname{gr} .(\mathbb{Q}-$ mod $)$ & $\mathcal{G}_{\text {top }}^{H}$ & $\mathrm{H}_{*} \mathcal{E}_{t}^{H}(X, Y)$ \\
\hline
\end{tabular}

For a ring $R$, let $\mathrm{Ch}(R$-mod) be the category of chain complexes of left $R$-modules and let gr. $(R$-mod) be the category of graded left $R$-modules. The category of chain complexes of $R$-modules has a model structure (sometimes called the projective model structure), where a map of chain complexes is a weak equivalence if it is a homology isomorphism and a fibration if it is a surjection. The cofibrations are level-wise split monomorphisms with cofibrant cokernel. For each $n \in \mathbb{Z}$, let $S^{n} R$ be the chain complex concentrated in degree $n$, where it takes value $R$. Let $D^{n} R$ be the chain complex with $R$ in degrees $n$ and $n-1$ and zeroes elsewhere, with the identity as the differential from degree $n$ to $n-1$. The projective model structure is cofibrantly generated with generating cofibrations the inclusions $S^{n-1} R \rightarrow D^{n} R$ and generating acyclic cofibrations the maps $0 \rightarrow D^{n} R$. See [Hov99, Section 2.3] for more details.

For a finite group $G$, let $\mathbb{Q} G$ be the rational group ring of $G$. This is a Hopf-algebra with the co-commutative coproduct $\Delta: \mathbb{Q} G \rightarrow \mathbb{Q} G \otimes \mathbb{Q} G$ induced by $g \mapsto g \otimes g$. For $\mathbb{Q} G$-chain complexes $X$ and $Y$ we have $X \otimes_{\mathbb{Q}} Y$, the tensor product of $X$ and $Y$ considered as objects of $\operatorname{Ch}\left(\mathbb{Q}\right.$-mod). For $n \in \mathbb{Z},\left(X \otimes_{\mathbb{Q}} Y\right)_{n}=$ $\oplus_{i+j=n} X_{i} \otimes_{\mathbb{Q}} Y_{j}$ and we define a $G$-action by $g \cdot(x \otimes y)=(g \cdot x) \otimes(g \cdot y)$. Hence $X \otimes_{\mathbb{Q}} Y$ is an object of $\mathrm{Ch}(\mathbb{Q} G$-mod $)$. That this product is associative and commutative follows from the corresponding properties for the tensor product of $\mathrm{Ch}(\mathbb{Q}$-mod) and the co-commutative Hopf-algebra structure on $\mathbb{Q} G$. The unit of this product is $S^{0} \mathbb{Q}$ equipped with trivial $G$-action. Furthermore, there is an internal homomorphism object, defined by $\operatorname{Hom}_{\mathbb{Q}}(X, Y)_{n}=\prod_{k} \operatorname{Hom}_{\mathbb{Q}}\left(X_{k}, Y_{n+k}\right)$, with $G$-action by conjugation and we have a natural isomorphism

$$
\operatorname{Ch}(\mathbb{Q} G \text {-mod })\left(X \otimes_{\mathbb{Q}} Y, Z\right) \cong \operatorname{Ch}(\mathbb{Q} G \text {-mod })\left(X, \operatorname{Hom}_{\mathbb{Q}}(Y, Z)\right) .
$$

Definition 4.1. For any $X \in \mathrm{Ch}(\mathbb{Q} G$-mod $)$, there is natural map of chain complexes $A v_{G}: X \rightarrow X^{G}$ defined by $A v_{G}(x)=|G|^{-1} \Sigma_{g \in G} g x$.

For a chain complex of $\mathbb{Q}$-modules $X$, let $\varepsilon^{*}(X)$ denote $X$ with the trivial action, an object of $\mathrm{Ch}(\mathbb{Q} G$-mod). This functor is the left adjoint of a strong symmetric 
monoidal adjoint pair (this terminology is defined later in this section)

$$
\varepsilon^{*}: \operatorname{Ch}(\mathbb{Q} \text {-mod }) \rightleftarrows \mathrm{Ch}(\mathbb{Q} G \text {-mod }):(-)^{G} .
$$

The right adjoint is the fixed point functor. We show that this is a Quillen pair by proving that the right adjoint preserves fibrations and weak equivalences. Take $f: X \rightarrow Y$ a surjection and let $y \in Y^{G}$; then there is an $x$ such that $f(x)=y$. Let $A v_{G}(x)=|G|^{-1} \Sigma_{g \in G} g x$; then since $A v_{G}(x) \in X^{G}$ and $f\left(A v_{G}(x)\right)=A v_{G}(f(x))=$ $A v_{G}(y)=y$, it follows that $f^{G}$ is surjective. That $(-)^{G}$ preserves weak equivalences is immediate: $H_{*}\left(X^{G}\right) \cong\left(H_{*} X\right)^{G}$, as we are working rationally. This implies that $\mathbb{Q}=\varepsilon^{*}(\mathbb{Q})$ is cofibrant as an object of $\mathrm{Ch}(\mathbb{Q} G$-mod $)$.

Definition 4.2. Let $\mathcal{C}$ be a cofibrantly generated model category, with a symmetric monoidal product $\otimes$, an internal function object $\operatorname{Hom}_{\mathcal{C}}(-,-)$ and a unit $I$. Then $\mathcal{C}$ satisfies the pushout product axiom if the following three conditions hold (see [SS00, Lemma 3.5(1)]), in which case $\mathcal{C}$ is called a monoidal model category:

1. If $f: A \rightarrow B$ and $g: C \rightarrow D$ are generating cofibrations, then the pushout product, $f \diamond g: B \otimes C \coprod_{A \otimes C} A \otimes D \rightarrow B \otimes D$, is a cofibration.

2. If $f$ is a generating cofibration and $g$ is a generating acyclic cofibration, then $f \diamond g$ is a weak equivalence.

3. If $X$ is a cofibrant object, then for any cofibrant replacement of the unit $\widehat{c} I \rightarrow I$ the induced map $X \otimes \widehat{c} I \rightarrow X \otimes I$ is a weak equivalence.

Let $Z$ be any object of $\mathcal{C}$ and let $P_{Z}$ be the set of maps of the form $\operatorname{Id}_{Z} \otimes f$, where $f$ is a generating acyclic cofibration. The class $P_{Z}$-cell ([Hov99, Definition 2.1.9]) is the collection of all maps formed by transfinite compositions of pushouts of maps of $P_{Z}$. The model category $\mathcal{C}$ satisfies the monoid axiom if for any object $Z$ the class $P_{Z}$-cell consists of weak equivalences (see [SS00, Lemma 3.5(2)]).

The pushout product axiom ensures that the monoidal product of a model category $\mathcal{C}$ induces a monoidal product on the homotopy category of $\mathcal{C}$. The monoid axiom (roughly speaking) ensures that there are model structures on the categories of $R$ algebras and $R$-modules, for $R$ a commutative monoid in $\mathcal{C}$.

Proposition 4.3. The tensor product and homomorphism object defined above gives the projective model structure on $\mathrm{Ch}(\mathbb{Q} G$-mod) the structure of a closed symmetric monoidal model category that satisfies the monoid axiom.

Proof. Let $f$ and $g$ be generating cofibrations, then $f \diamond g$ is an inclusion and the cokernel is $\mathbb{Q}(G \times G)$ (in some degree). This cokernel is cofibrant: it is isomorphic (as a $\mathbb{Q} G$-module) to $\bigoplus_{g \in G} \mathbb{Q} G$. For a generating cofibration $f$ and a generating acyclic cofibration $g, f \diamond g$ is a weak equivalence since both the domain and codomain are acyclic. Since the unit $\mathbb{Q}$ is cofibrant the last condition of the pushout product axiom holds automatically.

The projective model structure on $\mathrm{Ch}(\mathbb{Q}$-mod $)$ satisfies the monoid axiom; this is proved for a general ring in [Shi07a, Proposition 3.1]. In fact this proof also suffices to show that $\mathrm{Ch}(\mathbb{Q} G$-mod) satisfies the monoid axiom; we copy that proof with notation adjusted to our setting. 
The generating acyclic cofibrations for $\mathrm{Ch}\left(\mathbb{Q} G\right.$-mod) are the maps $0 \rightarrow D^{n}(\mathbb{Q} G)$, for $n$ an integer. Take any $Z \in \mathrm{Ch}(\mathbb{Q} G$-mod), then it is easy to check that $Z \otimes_{\mathbb{Q}} D^{n}(\mathbb{Q} G)$ is also acyclic. Then we note that $0 \rightarrow Z \otimes_{\mathbb{Q}} D^{n}(\mathbb{Q} G)$ is an injection and a homology isomorphism. Such maps are closed under pushouts and transfinite compositions - they are acyclic cofibrations in the injective model structure ([Hov99, Theorem 2.3.13]) on chain complexes of $\mathbb{Q} G$-modules. Hence the monoid axiom holds for $\mathrm{Ch}(\mathbb{Q} G$-mod).

The homotopy category of a pointed model category $\mathcal{C}$ supports a suspension functor $\Sigma$ with a right adjoint loop functor $\Omega$; see [Hov99, Section 6.1]. If these are inverse equivalences, then $\mathcal{C}$ is called a stable model category. All of the model categories that we use in this paper are stable model categories. An object $X$ of $\mathcal{C}$ is said to be compact if for any family of objects $\left\{Y_{i}\right\}_{i \in I}$, the canonical map $\oplus_{i \in I}\left[X, Y_{i}\right]^{\mathcal{C}} \rightarrow\left[X, \coprod_{i \in I} Y_{i}\right]^{\mathcal{C}}$, is an isomorphism. A stable model category $\mathcal{C}$ is said to be generated by a set of objects $\mathscr{P}$ if the smallest full triangulated subcategory of $\mathrm{Ho} \mathcal{C}$ (with shift and triangles induced from $\mathrm{Ho} \mathcal{C}$ ), that is closed under coproducts, is Ho $\mathcal{C}$ itself. By [SS03b, Lemma 2.2.1], if the set $\mathscr{P}$ consists of compact objects, then this statement is equivalent to the following: an object $X$ is trivial in the homotopy category if and only if $[P, X]_{*}^{\mathcal{C}}$ (graded maps in the homotopy category) is zero for each $P \in \mathscr{P}$.

Lemma 4.4. The model category $\mathrm{Ch}(\mathbb{Q} G$-mod) is generated by the compact object $\mathbb{Q} G$.

Proof. Let $X \in \mathrm{Ch}(\mathbb{Q} G$-mod $)$, then $[\mathbb{Q} G, X]_{*}^{\mathbb{Q} G} \cong[\mathbb{Q}, X]^{\mathbb{Q}} \cong H_{*}(X)$.

We now take the time to introduce the terminology of right modules over an enriched category and the notion of monoidal Quillen equivalences. We will use this machinery in several different settings and it provides the framework for our method of proof. Later we will use other model categories in place of $\mathrm{Ch}(\mathbb{Q}$-mod).

Definition 4.5. A $\mathrm{Ch}(\mathbb{Q}$-mod)-category is a category enriched over $\mathrm{Ch}(\mathbb{Q}$-mod) (see $[$ Kel05, Section 1.5]). A right module over a $\mathrm{Ch}(\mathbb{Q}$-mod)-category $\mathcal{E}$ is a contravariant enriched functor $M: \mathcal{E} \rightarrow \mathrm{Ch}(\mathbb{Q}$-mod); the category of such functors and enriched natural transformations is denoted by mod-E. The free module on an object $a$ of $\mathcal{E}$ is $F_{a}=\operatorname{Hom}_{\mathbb{Q}}(-, a)$.

Let $M$ be a right $\mathcal{E}$-module. Then for each object $a$ of $\mathcal{E}$ there is an object $M(a) \in \mathrm{Ch}(\mathbb{Q}$-mod). For a pair of objects $a, b \in \mathcal{E}$, we have a map in $\mathrm{Ch}(\mathbb{Q}$-mod $)$

$$
M_{a, b}: \mathcal{E}(a, b) \rightarrow \operatorname{Hom}_{\mathbb{Q}}(M(b), M(a)) .
$$

A more useful version is given in terms of the adjoint to $M_{a, b}$, the 'action map' $M(b) \otimes_{\mathbb{Q}} \mathcal{E}(a, b) \rightarrow M(a)$. An enriched natural transformation $f: M \rightarrow N$ is a collection of maps $f(a): M(a) \rightarrow N(a)$ compatible with these action maps. We can also form the category $\mathrm{H}_{*} \mathcal{E}$; this has the same object set as $\mathcal{E}$ and is enriched over graded $\mathbb{Q}$-modules, with morphism objects defined by $\left(\mathrm{H}_{*} \mathcal{E}\right)(a, b)=\mathrm{H}_{*}(\mathcal{E}(a, b))$.

The category of right modules over $\mathcal{E}$ has a model structure with weak equivalences and fibrations defined object-wise in $\mathrm{Ch}(\mathbb{Q}$-mod); see [SS03b, Subsection 3.3]. The collection of free modules is a generating set and these are cofibrant since the 
unit of $\mathrm{Ch}(\mathbb{Q}$-mod) is. The generating (acyclic) cofibrations of mod-E $\mathcal{E}$ have the form $A \otimes_{\mathbb{Q}} \operatorname{Id}_{F_{a}} \rightarrow B \otimes_{\mathbb{Q}} \operatorname{Id}_{F_{a}}$ (the object-wise tensor product) for $A \rightarrow B$ a generating (acyclic) cofibration of $\mathrm{Ch}(\mathbb{Q}$-mod).

Following [Day70, page 2] we define a symmetric monoidal enriched category as an enriched category $\mathcal{E}$, with an enriched functor $\otimes: \mathcal{E} \times \mathcal{E} \rightarrow \mathcal{E}$ satisfying associativity, unitary and symmetry conditions. Such a category has a 'unit object', which we denote by $I$. Thus for any two objects $a$ and $b$ of $\mathcal{E}$ we have an object $a \otimes b$ in $\mathcal{E}$, and for each quadruple $(a, b, c, d)$ of objects of $\mathcal{E}$ we have a map

$$
\mathcal{E}(a, c) \otimes_{\mathbb{Q}} \mathcal{E}(b, d) \rightarrow \mathcal{E}(a \otimes b, c \otimes d),
$$

which is compatible with the composition of $\mathcal{E}$. The associativity, unitary and symmetry conditions imply that for any quadruple $(a, b, c, d)$ we have isomorphisms as below, which are compatible with the composition of $\mathcal{E}$ :

$$
\begin{aligned}
\mathcal{E}((a \otimes b) \otimes c, d) & \cong \mathcal{E}(a \otimes(b \otimes c), d), \\
\mathcal{E}(d,(a \otimes b) \otimes c) & \cong \mathcal{E}(d, a \otimes(b \otimes c)), \\
\mathcal{E}(a \otimes I, d) & \cong \mathcal{E}(a, d), \\
\mathcal{E}(d, a \otimes I) & \cong \mathcal{E}(d, a), \\
\mathcal{E}(a \otimes b, d) & \cong \mathcal{E}(b \otimes a, d), \\
\mathcal{E}(d, a \otimes b) & \cong \mathcal{E}(d, b \otimes a) .
\end{aligned}
$$

By assuming that $\mathcal{E}$ is a symmetric monoidal enriched category (and that the collection of objects of $\mathcal{E}$ forms a set), we can put a symmetric monoidal structure on mod- $\mathcal{E}$ with unit the free module on $I: \mathcal{E}(-, I)$. The formula used is quite complicated, but it occurs often when constructing monoidal products. The right-hand side of the definition will be a coend, a particular form of a colimit and we give some details after the definition. Let $M$ and $N$ be two objects of mod- $\mathcal{E}$, then their box product, $M \square N$, is defined by the formula below.

$$
M \square N(a)=\int^{b, c} M(b) \otimes_{\mathbb{Q}} N(c) \otimes_{\mathbb{Q}} \mathcal{E}(a, b \otimes c) .
$$

If $F: \mathcal{C}^{o p} \times \mathcal{C} \rightarrow \mathrm{Ch}(\mathbb{Q}$-mod $)$ is a $\mathrm{Ch}(\mathbb{Q}$-mod)-enriched functor, where the objects of $\mathcal{C}$ form a set, then

$$
\int^{a} F(a, a)=\operatorname{coeq}\left(\coprod_{b, c \in \mathcal{C}} F(b, c) \otimes_{\mathbb{Q}} \mathcal{C}(c, b) \Longrightarrow \coprod_{d \in \mathcal{C}} F(d, d)\right) .
$$

The two maps are given by $\mathcal{C}(c, b)$ acting on either the first or second variable of $F$. We note that if $G$ is a right module over $\mathcal{C}$, then $\int^{a} G(a) \otimes_{\mathbb{Q}} \mathcal{C}(b, a) \cong G(b)$ and if we have a functor

$$
H: \mathcal{C}^{o p} \times \mathcal{C}^{o p} \times \mathcal{C} \times \mathcal{C} \rightarrow \mathrm{Ch}(\mathbb{Q}-\bmod ),
$$

then there is a canonical isomorphism $\int^{a} \int^{b} H(a, b, a, b) \cong \int^{b} \int^{a} H(a, b, a, b)$. Hence we allow ourselves to write $\int^{a, b} H(a, b, a, b)$ for either of these. The use of the functor $\otimes: \mathcal{E} \times \mathcal{E} \rightarrow \mathcal{E}$ is hidden in the definition of $\square$, but becomes clear when $M \square N$ is 
written in terms of a coequaliser of coproducts. For more information on enriched categories and coends; see [Bor94] or [Mac71].

The pushout product axiom and monoid axiom for $\mathrm{Ch}(\mathbb{Q}$-mod $)$ imply that they also hold for mod- $\mathcal{E}$. This routine statement is proved in [Bar08, Theorem 5.3.9], which also implies that the pushout product and monoid axioms hold in all the categories of right modules that we will encounter.

As well as enrichments, we can also consider tensorings and cotensorings over $\mathrm{Ch}(\mathbb{Q}$-mod). That is, $\mathcal{C}$ is tensored over $\mathrm{Ch}(\mathbb{Q}$-mod $)$ if it is equipped with a functor

$$
-\otimes-: \operatorname{Ch}(\mathbb{Q}-\bmod ) \times \mathcal{C} \rightarrow \mathcal{C},
$$

unit isomorphisms $\mathbb{Q} \otimes a \cong a$ and associativity isomorphisms

$$
\left(M \otimes_{\mathbb{Q}} N\right) \otimes a \cong M \otimes(N \otimes a) .
$$

Similarly, $\mathcal{C}$ is cotensored over $\operatorname{Ch}(\mathbb{Q}$-mod) if there is a functor

$$
\operatorname{Hom}(-,-): \operatorname{Ch}(\mathbb{Q}-\text { mod })^{o p} \times \mathcal{C} \rightarrow \mathcal{C}
$$

also satisfying unital and associativity conditions. Often a category $\mathcal{C}$ will be enriched, tensored and cotensored over $\mathrm{Ch}(\mathbb{Q}$-mod) all at once, whereupon for a chain complex $M$ and objects $a$ and $b$ of $\mathcal{C}$ we require isomorphisms of chain complexes as below that make all the various unital and associativity conditions compatible.

$$
\operatorname{Hom}_{\mathbb{Q}}(M, \mathcal{C}(a, b)) \cong \mathcal{C}(M \otimes a, b) \cong \mathcal{C}(a, \operatorname{Hom}(M, b)) .
$$

One important consequence of having all three of these structures linked by isomorphisms as above is that this ensures that tensor operation preserves colimits in both variables (similar statements then hold for the cotensor and enrichment).

Let $\mathcal{C}$ be a model category which is enriched, tensored and cotensored over the category $\mathrm{Ch}(\mathbb{Q}$-mod), with isomorphisms relating the three structures as above. Then $\mathcal{C}$ is said to be a $\mathrm{Ch}(\mathbb{Q}$-mod)-model category ([Hov99, Definition 4.2.18]) if whenever $f$ is a cofibration of $\mathcal{C}$ and $g$ is a cofibration of $\mathrm{Ch}(\mathbb{Q}$-mod), then $f \diamond g$ (the notation $\diamond$ is from Definition 4.2) is a cofibration of $\mathcal{C}$ that is acyclic if one of $f$ or $g$ is.

By definition a $\mathrm{Ch}(\mathbb{Q}$-mod)-model category is a $\mathrm{Ch}(\mathbb{Q}$-mod $)$-category. Note that $\mathrm{Ch}(\mathbb{Q} G$-mod $)$ is a $\mathrm{Ch}(\mathbb{Q}$-mod)-model category with tensor, cotensor and enrichment defined via the adjunction $\left(\varepsilon^{*},(-)^{G}\right)$. So for a $\mathbb{Q}$-chain complex $M$ and $\mathbb{Q} G$-chain complexes $X$ and $Y$, the tensor product is given by $M \otimes X=\varepsilon^{*}(M) \otimes_{\mathbb{Q}} X$, the cotensor by $\operatorname{Hom}_{\mathbb{Q}}\left(\varepsilon^{*}(M), X\right)$ and the enrichment by $\operatorname{Hom}_{\mathbb{Q}}(X, Y)^{G}$.

For $H$ a subgroup of $G$, let $N_{G} H$ be the normaliser of $H$ : the largest subgroup of $G$ which contains $H$ as a normal subgroup, we then define $W_{G} H=N_{G} H / H$, the Weyl group of $H$ in $G$. We write $\mathbb{Q} W_{G} H$-mod for the category of $\mathbb{Q}$-modules with a left action of $W_{G} H$.

Definition 4.6. Let $\mathcal{G}_{a, G}=\{\mathbb{Q}, \mathbb{Q} G, \mathbb{Q}(G \times G), \mathbb{Q}(G \times G \times G), \ldots\}$ and define $\mathcal{E}_{a, G}$ to be the $\mathrm{Ch}\left(\mathbb{Q}\right.$-mod)-category with object set $\mathcal{G}_{a, G}$ and $\mathrm{Ch}(\mathbb{Q}$-mod)-mapping object given by $\mathcal{E}_{a, G}(X, Y)=\operatorname{Hom}_{\mathbb{Q}}(X, Y)^{G}$. Now we define $\mathcal{G}_{a, G}^{H}=\mathcal{G}_{a, W_{G} H}$ and $\mathcal{E}_{a, G}^{H}=$ $\mathcal{E}_{a, W_{G} H}$. We will usually suppress the $G$ and reduce this notation to $\mathcal{G}_{a}^{H}$ and $\mathcal{E}_{a}^{H}$. 
Since the enrichment of $\mathrm{Ch}\left(\mathbb{Q} W_{G} H\right.$-mod) over $\mathrm{Ch}(\mathbb{Q}$-mod $)$ is defined in terms of the strong symmetric monoidal adjunction $\left(\varepsilon^{*},(-)^{G}\right)$, the result below follows by a routine argument.

Lemma 4.7. The category $\mathcal{E}_{a}^{H}$ is a symmetric monoidal $\mathrm{Ch}(\mathbb{Q}$-mod)-category.

The model categories $\mathrm{Ch}\left(\mathbb{Q} W_{G} H\right.$-mod $)$ and $\bmod -\mathcal{E}_{a}^{H}$ are Quillen equivalent by [SS03b, Theorem 3.9.3]; we describe the Quillen adjoint pair of this result. The proof that these are an equivalence is based on showing that the unit and counit of the derived adjunction are isomorphisms on the generators (the elements of $\mathcal{G}_{a}^{H}$ and the free modules). Let $X$ be an object of $\mathrm{Ch}\left(\mathbb{Q} W_{G} H\right.$-mod $)$, then consider the functor $\operatorname{Hom}_{\mathbb{Q}}(-, X)^{W_{G} H}: \mathcal{E}_{a}^{H} \rightarrow \mathrm{Ch}(\mathbb{Q}$-mod $)$. This functor is enriched over $\mathrm{Ch}(\mathbb{Q}$-mod $)$ and thus we have defined an object of $\bmod -\mathcal{E}_{a}^{H}$. Now let

$$
\underline{\operatorname{Hom}}\left(\mathcal{G}_{a}^{H},-\right): \operatorname{Ch}\left(\mathbb{Q} W_{G} H \text {-mod }\right) \rightarrow \bmod -\mathcal{E}_{a}^{H}
$$

be that functor which sends an object $X$ to the object $\operatorname{Hom}_{\mathbb{Q}}(-, X)^{W_{G} H}$. This functor has a left adjoint, $(-) \otimes_{\mathcal{E}_{a}^{H}} \mathcal{G}_{a}^{H}$, defined in terms of a coend. Let $M \in \bmod -\mathcal{E}_{a}^{H}$; then

$$
M \otimes_{\mathcal{E}_{a}^{H}} \mathcal{G}_{a}^{H}=\int^{a} M(a) \otimes a .
$$

Now we show that the Quillen equivalence between mod- $\mathcal{E}_{a}^{H}$ and $\operatorname{Ch}\left(\mathbb{Q} W_{G} H\right.$-mod $)$ respects the monoidal structures. We first need some terminology.

Definition 4.8. Let $L: \mathcal{C} \rightleftarrows \mathcal{C}^{\prime}: R$ be an adjunction between monoidal categories $(\mathcal{C}, \otimes, I)$ and $\left(\mathcal{C}^{\prime}, \otimes^{\prime}, I^{\prime}\right)$. Then $(L, R)$ is a strong monoidal adjunction if the left adjoint is strong monoidal: so $L A \otimes^{\prime} L B \cong L(A \otimes B)$, the units are related by an isomorphism $L I \cong I^{\prime}$ and $L$ satisfies the associativity and unital coherence conditions of [Hov99, Definition 4.1.2]. Let $(L, R)$ be an adjunction of monoidal model categories such that there is a natural map $R X \otimes R Y \rightarrow R\left(X \otimes^{\prime} Y\right)$ and a specified map $I \rightarrow R I^{\prime}$ which satisfy the associativity and unit conditions of [Bor94, Diagrams 6.27 and 6.28]. This implies that the left adjoint has a natural map $m: L(A \otimes B) \rightarrow L A \otimes^{\prime} L B$ and a map $L I \rightarrow I^{\prime}$. We say that such an adjunction, $(L, R)$, is a monoidal Quillen pair (also known as lax monoidal or weak monoidal) if whenever $A$ and $B$ are cofibrant the map $m$ is a weak equivalence and if for any cofibrant replacement $\widehat{c} I \rightarrow I$ the composite $L(\widehat{c} I) \rightarrow L I \rightarrow I^{\prime}$ is a weak equivalence.

If $(L, R)$ is a Quillen pair between monoidal model categories, such that $L$ is strong monoidal and the unit of the domain of $L$ is cofibrant, then $(L, R)$ is a monoidal Quillen pair. The conditions of a weak monoidal Quillen pair ensure that on homotopy categories the derived adjunction is strong monoidal. In general, a monoidal Quillen equivalence between monoidal model categories satisfying the monoid axiom induces a Quillen equivalence on the categories of algebras and modules; see [SSO3a, Theorem 3.12].

The adjoint pair $\left((-) \otimes_{\mathcal{E}_{a}^{H}} \mathcal{G}_{a}^{H}, \underline{\operatorname{Hom}}\left(\mathcal{G}_{a}^{H},-\right)\right)$, is a strong symmetric monoidal Quillen adjunction, we prove part of this below; see also [GS, Proposition 3.6]. Let 
$M$ and $N$ be $\mathcal{E}_{a}^{H}$-modules, then

$$
\begin{aligned}
(M \square N) \otimes_{\mathcal{E}_{a}^{H}} \mathcal{G}_{a}^{H} & =\int^{g} \int^{a, b}(M(a) \otimes N(b) \otimes \mathcal{E}(\mathcal{G})(g, a \otimes b)) \otimes g \\
& \cong \int^{a, b} M(a) \otimes N(b) \otimes\left(\int^{g} \mathcal{E}_{a}^{H}(g, a \otimes b) \otimes g\right) \\
& \cong \int^{a, b} M(a) \otimes N(b) \otimes(a \otimes b) \\
& \cong \int^{a, b}(M(a) \otimes a) \otimes(N(b) \otimes b) \\
& \cong \int^{a}\left((M(a) \otimes a) \otimes \int^{b}(N(b) \otimes b)\right) \\
& \cong\left(\int^{a} M(a) \otimes a\right) \otimes\left(\int^{b} N(b) \otimes b\right) \\
& =M \otimes_{\mathcal{E}_{a}^{H}} \mathcal{G}_{a}^{H} \otimes N \otimes \mathcal{E}_{a}^{H} \mathcal{G}_{a}^{H} .
\end{aligned}
$$

We summarise the above work in the following result which gathers all of the information of the category $\mathrm{Ch}\left(\mathbb{Q} W_{G} H\right.$-mod) into a category of modules over a $\mathrm{Ch}(\mathbb{Q}$-mod)-enriched category.

Theorem 4.9. There is a strong symmetric monoidal Quillen equivalence of symmetric monoidal model categories that satisfy the monoid axiom:

$$
(-) \otimes_{\mathcal{E}_{a}^{H}} \mathcal{G}_{a}^{H}: \bmod -\mathcal{E}_{a}^{H} \rightleftarrows \mathrm{Ch}\left(\mathbb{Q} W_{G} H \text {-mod }\right): \underline{\operatorname{Hom}}\left(\mathcal{G}_{a}^{H},-\right) .
$$

We now wish to repeat this operation for the model category of $G$-spectra and encode all of its information into a $\mathrm{Ch}(\mathbb{Q}$-mod)-category, this is a much more complicated task. Once we have achieved this, we can compare this enriched category to $\mathcal{E}_{a}^{H}$.

\section{Rational $G$-spectra and splitting}

We introduce a category of rational $G$-spectra and use idempotents of the rational Burnside ring in Corollary 5.7 to split this category into a product of model categories, each generated by a single object, indexed over the conjugacy classes of subgroups of $G$.

We also provide a version of this splitting in terms of modules over a ring spectrum (Proposition 5.11). We end this section with Theorem 5.14, which performs the analogue of Theorem 4.9 for $S_{H}$-mod. We need to use the category $S_{H}$-mod in this result for technical reasons, as we explain in Remark 5.15. We take our time in introducing $S_{H}$-mod as we need to understand the weak equivalences and generators of these split pieces and it is easier to do so first, and then move to modules over ring spectra.

The category of $G$-equivariant EKMM $S$-modules, $G \mathcal{M}$, is defined in [MM02, Chapter IV]. We refer to the objects of this category as $S$-modules or $G$-spectra or just spectra. Let $H$ be a subgroup of $G$ and $n \geqslant 0$, then for an $S$-module $X$, we 
have the homotopy groups $\pi_{n}^{H}(X)=\pi_{n}\left(X(0)^{H}\right)$ and $\pi_{-n}^{H}(X)=\pi_{n}\left(X\left(\mathbb{R}^{n}\right)^{H}\right)$. A map $f: X \rightarrow Y$ is called a $\pi_{*}$-isomorphism if $\pi_{n}^{H}(f)$ is an isomorphism for all integers $n$ and subgroups $H$ of $G$. See [MM02, Chapter IV, Theorem 2.9] for the following result.

Theorem 5.1. For $G$ a compact Lie group, there is a cofibrantly generated, proper, closed symmetric monoidal model structure on $G \mathcal{M}$ with weak equivalences the $\pi_{*}$ isomorphisms. Every object of this category is fibrant.

Let $E$ be a $G$-spectrum and let $X$ be a $G$-space; then we have a graded abelian group $\left[\Sigma^{\infty} X, E\right]_{*}^{G}$. This is the set of graded maps from the suspension spectrum of $X$ to $E$ in the homotopy category of $G$-spectra. We can think of this as a functor $E^{*}$ from the homotopy category of $G$-spaces to the category of graded abelian groups. The isomorphism classes of such functors as $E$ varies is the category of $G$-equivariant cohomology theories. One could equally well give a direct definition of a $G$-cohomology theory ([May96, Chapter XIII, Definition 1.1]) and then prove that the category of such objects is equivalent to the homotopy category of $G$-equivariant spectra. If $E^{*}(X)$ is a $\mathbb{Q}$-module for every space $X$, then we say that $E^{*}$ is a rational $G$-cohomology theory. A map $f$ is called a rational $\pi_{*}$-isomorphism (also called a rational equivalence or a $\pi_{*}^{\mathbb{Q}}$-isomorphism) if $\pi_{n}^{H}(f) \otimes \mathbb{Q}$ is an isomorphism for all integers $n$ and subgroups $H$ of $G$. We now give a result that summarises [Bar08, Section 2.2]. The homotopy category of the following model category is the category of rational $G$-equivariant cohomology theories.

Theorem 5.2. There is a cofibrantly generated, proper, closed symmetric monoidal model structure on the category of $G$-equivariant $S$-modules with weak equivalences the $\pi_{*}^{\mathbb{Q}}$-isomorphisms; we denote this model structure by $G \mathcal{M}_{\mathbb{Q}}$. Maps in the homotopy category of $G \mathcal{M}_{\mathbb{Q}}$ will be written $[X, Y]_{\mathbb{Q}}^{G}$ and these sets are always rational vector spaces. The fibrant objects are precisely those spectra with rational homotopy groups.

Recall [MM02, Chapter IV, Theorem 6.3] which states that for a cofibrant spectrum $E \in G \mathcal{M}$, there is an $E$-model structure on the category of $G$-equivariant $S$-modules with the same cofibrations as before and weak equivalences those maps $f$ such that $f \wedge \operatorname{Id}_{E}$ is a $\pi_{*}$-isomorphism. This model structure is called the Bousfield localisation of $G \mathcal{M}$ at $E$ and is written $L_{E} G \mathcal{M}$, the fibrant objects of this category are precisely the $E$-local objects. Note that in terms of model categories $L_{E \wedge F} G \mathcal{M}=L_{E} L_{F} G \mathcal{M}=L_{F} L_{E} G \mathcal{M}$; that is, the weak equivalences, cofibrations and fibrations are the same.

We construct $G \mathcal{M}_{\mathbb{Q}}$ by localising at $S_{\mathcal{M}}^{0} \mathbb{Q}$, a 'rational sphere spectrum'. This is a cofibrant spectrum such that the $S_{\mathcal{M}}^{0} \mathbb{Q}$-equivalences are the $\pi_{*}^{\mathbb{Q}}$-isomorphisms. This spectrum is constructed as follows, using $\widehat{c}$ to denote cofibrant replacement. Take $0 \rightarrow \oplus_{i} \mathbb{Z} \stackrel{f}{\rightarrow} \oplus_{j} \mathbb{Z} \rightarrow \mathbb{Q} \rightarrow 0$, a free resolution of $\mathbb{Q}$ as a $\mathbb{Z}$-module. Choose a map $g: \vee_{i} \widehat{c} S \rightarrow \vee_{j} \widehat{c} S$ such that $\pi_{0}^{G}(g)$ is given by $f \otimes \mathrm{Id}: \oplus_{i} \mathbb{Z} \otimes A(G) \rightarrow \oplus_{j} \mathbb{Z} \otimes A(G)$. The spectrum $S_{\mathcal{M}}^{0} \mathbb{Q}$ is then defined to be the cofibre of $g$. With these definitions we can now give [Bar08, Theorem 3.2.4], which we will use to split the category of rational $G$-spectra into more manageable pieces. 
Theorem 5.3. Let $\left\{E_{i}\right\}_{i \in I}$ be a finite collection of cofibrant orthogonal $G$-spectra or $G$-spaces. If $E_{i} \wedge E_{j}$ is rationally acyclic for $i \neq j$ and $\bigvee_{i \in I} E_{i}$ is rationally equivalent to $S$, then we have a strong symmetric monoidal Quillen equivalence

$$
\Delta: G \mathcal{M}_{\mathbb{Q}} \rightleftarrows \prod_{i \in I} L_{E_{i}} G \mathcal{M}_{\mathbb{Q}}: \prod
$$

The left adjoint takes a $G$-spectrum $X$ to the constant collection of $X$ in each factor. The right adjoint takes the collection $\left\{Y_{i}\right\}_{i \in I}$ to the G-spectrum $\prod_{i \in I} Y_{i}$.

An important step in the proof of this theorem is showing that if $X$ is $E_{i}$-local then $E_{j} \wedge X \rightarrow *$ is a rational equivalence whenever $i \neq j$. We will need this later and in fact this result can be deduced from the above theorem.

Take $X$ an $E_{i}$-local $G$-spectrum, then the collection $\left\{X_{i}\right\}_{i \in I}$ defined by $X_{i}=X$ and $X_{j}=*$ whenever $i \neq j$, is a fibrant object of $\prod_{i \in I} L_{E_{i}} G \mathcal{M}_{\mathbb{Q}}$. Since $\Delta$ preserves all weak equivalences and $(\Delta, \Pi)$ is a Quillen equivalence, it follows that the counit is a weak equivalence. Hence $X$ is $E_{j}$-equivalent to $*$ whenever $i \neq j$.

The Burnside ring of $G, A(G)$, is the Grothendieck ring of finite $G$-sets and is isomorphic to $[S, S]_{*}^{G}$. Since $G$ is finite, tom Dieck's isomorphism (see [LMSM86, Chapter V, Lemma 2.10]) specifies an isomorphism $A(G) \otimes \mathbb{Q} \cong \prod_{(H) \leqslant G} \mathbb{Q}$. Thus, for each conjugacy class of subgroups, $(H) \leqslant G$, there is an idempotent $e_{H} \in A(G) \otimes \mathbb{Q}$ given by projection onto factor $(H)$. Let $\widehat{f}_{\mathbb{Q}}$ denote a fibrant replacement in $G \mathcal{M}_{\mathbb{Q}}$; then $A(G) \otimes \mathbb{Q} \cong\left[\widehat{f}_{\mathbb{Q}} S, \widehat{f}_{\mathbb{Q}} S\right]^{G}$. Given an idempotent $e$ in the rational Burnside ring we write $e S$ for the homotopy colimit (telescope) of $S \rightarrow \widehat{f}_{\mathbb{Q}} S \stackrel{f}{\rightarrow} \widehat{f}_{\mathbb{Q}} S \stackrel{f}{\rightarrow} \cdots$, for some representative $f$ of $e$. Using the diagram $X \rightarrow X \wedge \widehat{f}_{\mathbb{Q}} S \stackrel{\operatorname{Id} \wedge f}{\longrightarrow} X \wedge \widehat{f}_{\mathbb{Q}} S \stackrel{\text { Id } \wedge f}{\longrightarrow} \cdots$, we construct $e X$ for any spectrum $X$. The map $X \rightarrow X \wedge \widehat{f}_{\mathbb{Q}} S$ is a $\pi_{*}^{\mathbb{Q}}$-isomorphism. Hence $e$ (or rather $\operatorname{Id}_{X} \wedge f$ ) induces a self-map of $\pi_{*}^{H}(X) \otimes \mathbb{Q}$; we write this map as $\iota_{H}^{*}(e)_{*}$. Homotopy groups and idempotents commute in the sense that the canonical $\operatorname{map} \iota_{H}^{*}(e)_{*} \pi_{*}^{H}(X) \otimes \mathbb{Q} \rightarrow \pi_{*}^{H}(e X) \otimes \mathbb{Q}$ is an isomorphism.

Definition 5.4. For a group $G$, with subgroups $H$ and $K$, we say that $K$ is subconjugate to $H$ if the $G$-conjugacy class of $K$ contains a subgroup of $H$, we write $K \leqslant_{G} H$. In turn, $K$ is strictly subconjugate to $H$ if the $G$-conjugacy class of $K$ contains a strict subgroup of $H$; the notation for this is $K<_{G} H$.

Definition 5.5. A set of subgroups of $G$ is called a family if it is closed under conjugation and taking subgroups. For each family $\mathcal{F}$ there is a $G$-CW complex $E \mathcal{F}$ which satisfies the universal property: $E \mathcal{F}^{H}$ is contractible for $H \in \mathcal{F}$ and is empty otherwise. The cofibre of the projection map $E \mathcal{F}_{+} \rightarrow S^{0}$ is denoted by $\widetilde{E} \mathcal{F}$.

Take $H$ a subgroup of $G$, then we have a pair of families of subgroups of $G$ : $[\leqslant G H$ - the family of all subgroups of $G$ which are subconjugate to $H$ and $\left[<_{G} H\right]$ - the family of all subgroups of $G$ which are strictly subconjugate to $H$. We can then form $G$-CW complexes $E\left[\leqslant_{G} H\right]_{+}$and $E\left[<_{G} H\right]_{+}$. There is a map $E\left[<_{G} H\right]_{+} \rightarrow E\left[\leqslant_{G} H\right]_{+}$; we call the cofibre of this map $E\langle H\rangle$. 
Note that since $E\left[<_{G} H\right]_{+}$and $E\left[\leqslant_{G} H\right]_{+}$are cofibrant as $G$-spaces, the space $E\langle H\rangle$ is also cofibrant as a $G$-space. We can also describe $E\langle H\rangle$ as

$$
E\left[\leqslant_{G} H\right]_{+} \wedge \widetilde{E}\left[<_{G} H\right] .
$$

Since geometric fixed point functors preserve cofibre sequences, the spectrum $\Phi^{K}\left(\Sigma^{\infty} E\langle H\rangle\right)$ is contractible unless $(K)=(H)$, whence it is non-equivariantly rationally equivalent to $S$. The following is a standard result proved by looking at geometric fixed points (see [Bar08, Lemma 3.4.11]).

Lemma 5.6. Let $e_{\left[\leqslant_{G} H\right]}=\Sigma_{(K) \leqslant H} e_{K}$ and $e_{\left[{ }_{G} H\right]}=\Sigma_{(K)<H} e_{K}$. Then there are zigzags of rational $\pi_{*}$-isomorphisms between $E\left[\leqslant_{G} H\right]_{+}$and $e_{\left[\leqslant_{G} H\right]} S$ and similarly so for $E\left[<_{G} H\right]_{+}$and $e_{\left[<_{G} H\right]} S$. Furthermore, $E\langle H\rangle$ is rationally equivalent to $e_{H} S$.

From this it follows that a map $f: X \rightarrow Y$ in $G \mathcal{M}_{\mathbb{Q}}$ is a rational $E\langle H\rangle$-equivalence if and only if $e_{H} f: e_{H} X \rightarrow e_{H} Y$ is a rational equivalence. We can now apply the splitting theorem using the set of objects $E\langle H\rangle$ as $H$ runs over a set of representatives for the conjugacy classes of subgroups of $G$. Since $E\langle H\rangle$ is rationally equivalent to $e_{H} S$ it follows that $\bigvee_{(H) \leqslant G} E\langle H\rangle$ is rationally equivalent to $S$ and $E\langle H\rangle \wedge E\langle K\rangle$ is rationally acyclic whenever $H$ and $K$ are not conjugate.

Corollary 5.7. There is a strong symmetric monoidal Quillen equivalence between the category of rational $G$-spectra and the product of the categories $L_{E\langle H\rangle} G \mathcal{M}_{\mathbb{Q}}$, as $H$ runs over the set of conjugacy classes of subgroups of $G$.

$$
\Delta: G \mathcal{M}_{\mathbb{Q}} \rightleftarrows \prod_{(H) \leqslant G} L_{E\langle H\rangle} G \mathcal{M}_{\mathbb{Q}}: \prod
$$

Lemma 5.8. There is an equality of model structures:

$$
\left.L_{E\langle H\rangle} G \mathcal{M}_{\mathbb{Q}}=L_{E\langle H\rangle} L_{E[\leqslant G H}\right]_{+} G \mathcal{M}_{\mathbb{Q}},
$$

that is to say, the weak equivalences, cofibrations and fibrations agree.

Proof. The cofibrations of these two model structures agree by definition. The map $\widetilde{E}\left[\leqslant_{G} H\right] \rightarrow *$ is a rational $E\langle H\rangle$ equivalence. Hence, considering the cofibre sequence which defines $\widetilde{E}\left[\leqslant_{G} H\right]$ we have a rational equivalence $E\left[\leqslant_{G} H\right]_{+} \wedge E\langle H\rangle \rightarrow E\langle H\rangle$. It follows that a rational $E\left[\leqslant_{G} H\right]_{+} \wedge E\langle H\rangle$-equivalence is a rational $E\langle H\rangle$-equivalence, so the weak equivalences of $L_{E\langle H\rangle} G \mathcal{M}_{\mathbb{Q}}$ and $L_{E\langle H\rangle} L_{E\left[{ }_{G} H\right]_{+}} G \mathcal{M}_{\mathbb{Q}}$ agree.

By [MM02, IV, Proposition 6.7], the weak equivalences of $L_{E\left[\leqslant_{G} H\right]_{+}} G \mathcal{M}_{\mathbb{Q}}$ are those maps $f$ such that $\pi_{*}^{K}(f) \otimes \mathbb{Q}$ is an isomorphism for all $K \leqslant_{G} H$.

Lemma 5.9. A map $f$ in $L_{E\langle H\rangle} G \mathcal{M}_{\mathbb{Q}}$ is a weak equivalence if and only if the induced map of homotopy groups $\iota_{H}^{*}\left(e_{H}\right)_{*} \pi_{*}^{H}(f) \otimes \mathbb{Q}$ is a isomorphism. Hence $G / H_{+}$is a compact generator for $L_{E\langle H\rangle} G \mathcal{M}_{\mathbb{Q}}$.

Proof. Lemma 5.8 shows that $f$ is a weak equivalence if and only if

$$
\iota_{K}^{*}\left(e_{H}\right)_{*} \pi_{*}^{K}(f) \otimes \mathbb{Q}
$$

is an isomorphism for all $K \leqslant_{G} H$. For any $G$-spectrum $X$, the set $\pi_{*}^{K}(X) \otimes \mathbb{Q}$ is a module over $A(K) \otimes \mathbb{Q}$. The rational Burnside ring of $G$ acts on $\pi_{*}^{K}(X) \otimes \mathbb{Q}$ via the 
restriction map $\iota_{K}^{*}: A(G) \otimes \mathbb{Q} \rightarrow A(K) \otimes \mathbb{Q}$ induced from the inclusion $\iota: K \rightarrow G$. Now note that if $K$ is a strict subgroup of $H$, then $\iota_{K}^{*}\left(e_{H}\right)=0$; hence for any map $f, \iota_{K}^{*}\left(e_{H}\right)_{*} \pi_{*}^{K}(f) \otimes \mathbb{Q}$ will be an isomorphism. This proves the first statement.

For any $G$-spectrum $X, e_{H} \pi_{*}^{H}(X) \otimes \mathbb{Q}$ is isomorphic to $\pi_{*}^{H}\left(\widehat{f}_{H} X\right)$, where $\widehat{f}_{H} X$ is the fibrant replacement of $X$ in $L_{E\langle H\rangle} G \mathcal{M}_{\mathbb{Q}}$; hence $\widehat{f}_{H} X$ is $S_{\mathcal{M}}^{0} \mathbb{Q} \wedge E\langle H\rangle$-local. In turn, $\pi_{*}^{H}\left(\widehat{f}_{H} X\right) \cong\left[G / H_{+}, \widehat{f}_{H} X\right]_{*}^{G}$, which is isomorphic to $\left(\left[G / H_{+}, X\right]_{\mathbb{Q}}^{G \mid H}\right)_{*}$, the set of graded maps in the homotopy category of $L_{E\langle H\rangle} G \mathcal{M}_{\mathbb{Q}}$. We have already shown that $X$ is trivial in Ho $L_{E\langle H\rangle} G \mathcal{M}_{\mathbb{Q}}$ if and only if $e_{H} \pi_{*}^{H}(X) \otimes \mathbb{Q}=0$. Now we know that $X$ is trivial in Ho $L_{E\langle H\rangle} G \mathcal{M}_{\mathbb{Q}}$ if and only if $\left(\left[G / H_{+}, X\right]_{\mathbb{Q}}^{G \mid H}\right)_{*}=0$. Hence $G / H_{+}$ is a generator; it is compact since $G / H_{+}$is a compact space.

Our next task is to obtain a version of $L_{E\langle H\rangle} G \mathcal{M}_{\mathbb{Q}}$ with every object fibrant (see Remark 5.15).

Lemma 5.10. There is an $S_{\mathcal{M}}^{0} \mathbb{Q} \wedge E\langle H\rangle$-local commutative $S$-algebra $S_{H}$ whose unit map is a rational $E\langle H\rangle$-equivalence. Furthermore, every $S_{H}$-module is $S_{\mathcal{M}}^{0} \mathbb{Q} \wedge E\langle H\rangle$ local.

Proof. This result is an application of [EKMM97, Chapter VIII, Theorem 2.2], which is easily adapted to an equivariant setting. We use the cell object $S_{\mathcal{M}}^{0} \mathbb{Q} \wedge E\langle H\rangle$ to create a commutative cell $S$-algebra $S_{H}$ which is the $S_{\mathcal{M}}^{0} \mathbb{Q} \wedge E\langle H\rangle$-localisation of $S$. By construction, the unit map $S \rightarrow S_{H}$ is a rational $E\langle H\rangle$-equivalence; hence $S_{\mathcal{M}}^{0} \mathbb{Q} \wedge E\langle H\rangle$ is $\pi_{*}$-isomorphic to $S_{H} \wedge S_{\mathcal{M}}^{0} \mathbb{Q} \wedge E\langle H\rangle$. Since $S_{H}$ is $S_{\mathcal{M}}^{0} \mathbb{Q}$-local, it has rational homotopy groups; thus there is a zig-zag of weak equivalences $S_{\mathcal{M}}^{0} \mathbb{Q} \wedge S_{H} \leftarrow$ $\widehat{c} S \wedge S_{H} \rightarrow S_{H}$. Equally, $S_{H}$ is weakly equivalent to $S_{H} \wedge \bigvee_{(K)} E\langle K\rangle$. Since $S_{H}$ is $E\langle H\rangle$-local, $S_{H} \wedge E\langle K\rangle$ is acyclic whenever $(H) \neq(K)$ (as noted above, this is part of the proof of Theorem 5.3). It follows that $S_{H} \wedge \bigvee_{(K)} E\langle K\rangle$ is weakly equivalent to $S_{H} \wedge E\langle H\rangle$. Thus $S_{H}$ is $\pi_{*}$-isomorphic to $S_{\mathcal{M}}^{0} \mathbb{Q} \wedge E\langle H\rangle$. The rest of the result is standard; see [Ada74, 13.1].

Proposition 5.11. The adjoint pair of the free $S_{H}$-module functor and the forgetful functor

$$
S_{H} \wedge(-): L_{E\langle H\rangle} G \mathcal{M}_{\mathbb{Q}} \rightleftarrows S_{H}-\bmod : U
$$

is a strong symmetric monoidal Quillen equivalence.

Proof. This is easy to prove. The two points to note are: an $S_{\mathcal{M}}^{0} \mathbb{Q} \wedge E\langle H\rangle$-equivalence between $S_{\mathcal{M}}^{0} \mathbb{Q} \wedge E\langle H\rangle$-local objects is a $\pi_{*}$-isomorphism and the unit map of $S_{H}$ is an $S_{\mathcal{M}}^{0} \mathbb{Q} \wedge E\langle H\rangle$-equivalence.

We now fix a cofibrant replacement of the suspension spectrum of $G / H_{+}$. We call this $\widehat{c} G / H_{+}$. One example is given by $S \wedge \mathscr{L} \mathbb{L} \Sigma^{\infty} G / H_{+}$; see [MM02, Chapter IV, Proposition 2.1].

Lemma 5.12. The object $\left(\widehat{c} G / H_{+}\right) \wedge S_{H}$ is a G-compact, cofibrant and fibrant generator of $S_{H}$-mod.

Proof. Every object of $S_{H}$-mod is fibrant and since $\widehat{c} G / H_{+}$is a cofibrant spectrum, $\left(\widehat{c} G / H_{+}\right) \wedge S_{H}$ is cofibrant in $S_{H}$-mod. This object is $G$-compact since the right 
adjoint $U$ commutes with filtered colimits and $G / H_{+}$is a $G$-compact $G$-spectrum. Since $G / H_{+}$generates $L_{E\langle H\rangle} G \mathcal{M}_{\mathbb{Q}}$, which is Quillen equivalent to $S_{H}$-mod, it follows that $S_{H}$-mod is generated by $\left(\widehat{c} G / H_{+}\right) \wedge S_{H}$.

Now we perform the analogue of Theorem 4.9 for $S_{H}$-mod. Recall the positive model structure as defined on symmetric spectra (and other categories of diagram spectra), written $S p_{+}^{\Sigma}$, from [MMSS01, Theorem 14.1]. The positive model structure has the same weak equivalences as $S p^{\Sigma}$ but the unit is no longer cofibrant. The identity functor is the left adjoint of a Quillen equivalence from $S p_{+}^{\Sigma}$ to $S p^{\Sigma}$. The adjunction $\left(\mathbb{N}, \mathbb{N}^{\#}\right)$ below, is only a Quillen pair when we use the positive model structure on equivariant orthogonal spectra $\left(G \mathscr{I}_{S_{+}^{U}}\right)$. Hence all the other categories below must be given their positive model structures.

We must first prove that $S_{H}$-mod is an $S p_{+}^{\Sigma}$-model category. We do so by constructing a strong symmetric monoidal Quillen adjunction with left adjoint mapping from $S p_{+}^{\Sigma}$ to $S_{H}$-mod. To do so we must be careful about the change of universe functors since we need these to be both strong monoidal and compatible with the model structures. To solve this we pass through equivariant orthogonal spectra, which are also defined in [MM02]. Thus we have the following unwieldy series of adjoint pairs.

The adjunction of geometric realisation and the singular complex functor (between simplicial sets and topological spaces) induces the Quillen equivalence below, where $S p^{\Sigma}(\mathrm{Top})_{+}$is the category of symmetric spectra of topological spaces with the positive model structure.

$$
|-|: S p_{+}^{\Sigma} \rightleftarrows S p^{\Sigma}(\mathrm{Top})_{+}: \text {Sing }
$$

One can then prolong to the positive model structure on orthogonal spectra (indexed on the universe $\mathbb{R}^{\infty}$ ), which we write as $\mathscr{I} \mathscr{S}_{+}^{\mathbb{R}^{\infty}}$, using the Quillen equivalence of [MMSS01, Theorem 10.4].

$$
\mathbb{P}: S p^{\Sigma}(\mathrm{Top})_{+} \rightleftarrows \mathscr{I} \mathscr{S}_{+}^{\mathbb{R}_{+}^{\infty}}: \mathbb{U}
$$

The trivial action and fixed point adjunction ([MM02, Chapter V, Section 3]) move us to $G$-equivariant orthogonal spectra indexed on a trivial universe.

$$
\varepsilon^{*}: \mathscr{I}_{+}^{\mathbb{R}_{+}^{\infty}} \rightleftarrows G \mathscr{I} \mathscr{S}_{+}^{\mathbb{R}^{\infty}}:(-)^{G}
$$

We apply change of universe functors to move to a complete universe $U$, using the notation of [MM02, Chapter V, Proposition 3.4].

$$
i_{*}: G \mathscr{I}_{+}^{\mathbb{R}_{+}^{\infty}} \rightleftarrows G \mathscr{I}_{+}^{U}: i^{*}
$$

We can relate the above to EKMM spectra using the Quillen equivalence of [MM02, Chapter IV, Theorem 1.1].

$$
\mathbb{N}: G \mathscr{I}_{+}^{U} \rightleftarrows G \mathcal{M}^{U}: \mathbb{N}^{\#}
$$

Then we make use of the free $S_{H}$-module functor.

$$
-\wedge S_{H}: G \mathcal{M}^{U} \rightleftarrows S_{H} \text {-mod: } U
$$

Since each of these adjoint pairs is strong symmetric monoidal, it follows that $S_{H}$-mod is an $S p_{+}^{\Sigma}$-model category. The enrichment is given by the following formula: Let $X$ 
and $Y$ be $S_{H}$-modules, then $\operatorname{Hom}(X, Y)=\operatorname{Sing} \mathbb{U}\left(i^{*} \mathbb{N} \# F_{S_{H}}(X, Y)\right)^{G}$ is the symmetric spectrum object of functions from $X$ to $Y$. That is, one takes the function spectrum of $S_{H}$-modules, applies the functor $\mathbb{N}^{\#}$, moves to a trivial universe $\left(i^{*}\right)$, takes $G$-fixed points, moves down to symmetric spectra of topological spaces and applies the singular complex functor to get to $S p_{+}^{\Sigma}$. This construction comes equipped with a natural isomorphism $\pi_{*}(\underline{\operatorname{Hom}}(X, Y)) \cong[X, Y]_{*}^{S_{H}}$.

Definition 5.13. Let $\mathcal{G}_{t o p}^{H}$ be the set of all smash products (in the category of $S_{H^{-}}$ modules) of $\left(\widehat{c} G / H_{+}\right) \wedge S_{H}$. We include $S_{H}$ as the zero-fold smash product. Let $\mathcal{E}_{t o p}^{H}$ be the $S p_{+}^{\Sigma}$-enriched category on the objects of $\mathcal{G}_{\text {top }}^{H}$.

Since the enrichment of $S_{H}$-mod over $S p_{+}^{\Sigma}$ is defined in terms of a (series of) strong symmetric monoidal adjunctions, it is routine to prove that $\mathcal{E}_{\text {top }}^{H}$ is a symmetric monoidal enriched category. With the exception of the unit, all objects of $\mathcal{G}_{\text {top }}^{H}$ are cofibrant and all objects are fibrant. We replace the category of $S_{H}$-modules by the Quillen equivalent category of modules over $\mathcal{E}_{\text {top }}^{H}$; see also [GS, Proposition 4.1]. Thus we have encoded $S_{H}$-mod in terms of a symmetric spectrum-enriched category. The adjunction below is analogous to the functors of Theorem 4.9.

Theorem 5.14. The adjoint pair

$$
(-) \wedge_{\mathcal{E}_{\text {top }}^{H}} \mathcal{G}_{\text {top }}^{H}: \bmod -\mathcal{E}_{\text {top }}^{H} \rightleftarrows S_{H} \text {-mod: } \underline{\operatorname{Hom}}\left(\mathcal{G}_{\text {top }}^{H},-\right)
$$

is a strong symmetric monoidal Quillen equivalence.

Proof. If $\sigma \in \mathcal{G}_{\text {top }}^{H}$ is not $S_{H}$, then it is cofibrant, so $\underline{\operatorname{Hom}}(\sigma,-)$ preserves fibrations and all weak equivalences when considered as a functor from $S_{H}$-mod to $S p_{+}^{\Sigma}$. The functor $\operatorname{Hom}\left(S_{H},-\right)$ preserves fibrations and all weak equivalences (since every object of $S_{H}$-mod is fibrant); hence the above adjunction is a Quillen pair.

Following the proof of [SSO3b, Theorem 3.9.3], we prove that the unit and counit of the derived adjunction are weak equivalences. It suffices to do so on the generators which are the free modules and the elements of $\mathcal{G}_{\text {top }}^{H}$. The free modules $F_{\sigma}=\operatorname{Hom}(-, \sigma)$ are not cofibrant. However, if we let $\widehat{c} \mathbb{S}$ be a cofibrant replacement of $\mathbb{S}$ (the sphere spectrum in $S p_{+}^{\Sigma}$ ) then $\widehat{c} \mathbb{S} \wedge F_{\sigma}$ is a cofibrant replacement of $F_{\sigma}$. The left derived functor, $(-) \wedge_{\mathcal{E}_{\text {top }}^{H}}^{L} \mathcal{G}_{\text {top }}^{H}$, takes $\widehat{c} \mathbb{S} \wedge F_{\sigma}$ to $\widehat{c} \mathbb{S} \wedge \sigma$. Since $\sigma$ is either $S_{H}$ or cofibrant, this is weakly equivalent to $\sigma$. The right adjoint preserves all weak equivalences and it follows that the unit and counit of the derived adjunction are weak equivalences. Hence we have a Quillen equivalence; that this is a strong symmetric monoidal follows by the same arguments as for the proof of Theorem 4.9.

Remark 5.15. In order to know that the above is a strong monoidal Quillen equivalence we need to know that $\mathcal{G}_{\text {top }}^{H}$ is closed under the smash product, that every object is fibrant and that every non-unit object is cofibrant. In a general model category there is no reason to expect that the smash product of fibrant objects will be fibrant. Hence we use the category of $S_{H}$-modules in EKMM $S$-modules, where every object is fibrant. 


\section{From $\mathcal{E}_{\text {top }}^{H}$ to $\mathcal{E}_{t}^{H}$}

We now have two enriched categories that we wish to compare: $\mathcal{E}_{\text {top }}^{H}$ and $\mathcal{E}_{a}^{H}$. The difficulty is that the first is enriched over (positive) symmetric spectra and the second is enriched over rational chain complexes. Theorem 6.5 creates a new category $\mathcal{E}_{t}^{H}$ from $\mathcal{E}_{\text {top }}^{H}$. These categories have the same set of objects: $\mathcal{G}_{\text {top }}^{H}$, but $\mathcal{E}_{t}^{H}$ is enriched over $\operatorname{Ch}\left(\mathbb{Q}\right.$-mod). The categories of modules mod- $\mathcal{E}_{t}^{H}$ and $\bmod -\mathcal{E}_{\text {top }}^{H}$ are Quillen equivalent as symmetric monoidal model categories. Furthermore, this construction comes with an isomorphism of monoidal $\mathrm{Ch}\left(\mathbb{Q}\right.$-mod)-enriched categories between $\mathrm{H}_{*} \mathcal{E}_{t}^{H}$ and $\pi_{*} \mathcal{E}_{\text {top }}^{H}$.

This theorem allows us to move from a topological setting to an algebraic setting, while preserving monoidal structures and also keeping control over the homology of the new category. In terms of [SS03b, Example 5.1.2] (see Section 2), this theorem corresponds to the zig-zag of Quillen equivalences between mod-H$\underline{A}$ and $\bmod -\underline{A}$. However this zig-zag passes through a model category without a monoidal product and so one cannot use it to to understand monoidal structures. Additionally, this zig-zag only exists when the homotopy groups of the $S p^{\Sigma}$-enriched category $\mathrm{H} \underline{A}$ are concentrated in degree zero, whereas our theorem has no such requirement.

Theorem 6.5 is similar to [Shi07a, Corollary 2.16], which is stated below. Recall that a stable model category is called rational if the set of maps in the homotopy category between any two objects forms a rational vector space (being stable implies that such sets are always abelian groups).

Proposition 6.1. Let $\mathcal{C}$ be a rational stable model category, with a set $\mathcal{G}$ of compact generators, which is Quillen equivalent to a $S p^{\Sigma}$-enriched model category. Then there $a$ is category $\mathcal{A}$, which is enriched over rational chain complexes and a chain of Quillen equivalences between $\mathcal{C}$ and the category of right $\mathcal{A}$-modules. The objects of $\mathcal{A}$ correspond to the objects in $\mathcal{G}$ and there is an isomorphism of graded $\mathbb{Q}$-categories between the homology category $\mathrm{H}_{*} \mathcal{A}$ and the full graded subcategory of $\operatorname{Ho}(\mathcal{C})$ with objects $\mathcal{G}$.

We introduce the Quillen pairs and model categories from [Shi07a] that we will need for our construction. Note that the functor $L$ below does not have a simple description so we concentrate on the remaining functors.

As well as $S p^{\Sigma}$, we also use $S p^{\Sigma}(\mathrm{s} \mathbb{Q}$-mod), symmetric spectra in simplicial $\mathbb{Q}$-modules; see [Hov01]. For symmetric spectra in simplicial sets, suspension is given in terms of $S^{1}$, for $S p^{\Sigma}\left(\mathrm{s} \mathbb{Q}\right.$-mod) suspension is defined using the object $\widetilde{\mathbb{Q}} S^{1}$, which we define below. We also use $S p^{\Sigma}\left(\mathrm{Ch}(\mathbb{Q} \text {-mod })_{+}\right)$, symmetric spectra in non-negatively graded chain complexes with suspension object $\mathbb{Q}[1]$, the chain complex consisting of $\mathbb{Q}$ in degree 1 . More generally we have $\mathbb{Q}[n]$, for $n$ an integer, which consists of $\mathbb{Q}$ in degree $n$.

The reduced free simplicial $\mathbb{Q}$-module functor from simplicial sets to simplicial $\mathbb{Q}$-modules induces strong symmetric monoidal Quillen adjunction below. Recall that reduced means that simplices of the form $0 \cdot x$ and $q \cdot *$ are identified with the basepoint.

$$
\widetilde{\mathbb{Q}}: S p^{\Sigma} \rightleftarrows S p^{\Sigma}(\mathrm{s} \mathbb{Q}-\bmod ): U
$$


Normalisation defines a functor $N: \mathrm{s} \mathbb{Q}-\bmod \rightarrow \mathrm{Ch}(\mathbb{Q} \text {-mod })_{+}$; in fact, $N$ is the right adjoint of monoidal Quillen equivalence between these two categories. The functor $N$ is not strong monoidal, so $N$ does not directly induce a functor on the level of symmetric spectra. Take an object $X$ of $S p^{\Sigma}(\mathrm{s} \mathbb{Q}-\bmod )$ and apply $N$ levelwise to $X$. There is an isomorphism $\mathbb{Q}[1] \rightarrow N\left(\widetilde{\mathbb{Q}} S^{1}\right)$; hence we have maps

$$
\phi_{n}: \mathbb{Q}[n] \stackrel{\cong}{\rightrightarrows} N\left(\widetilde{\mathbb{Q}} S^{1}\right)^{\otimes_{n}} \rightarrow N\left(\left(\widetilde{\mathbb{Q}} S^{1}\right)^{\otimes_{n}}\right),
$$

which we use to obtain a collection of maps $\mathbb{Q}[n] \otimes N X_{m} \rightarrow N X_{n+m}$. This information assembles to give an object of $S p^{\Sigma}\left(\mathrm{Ch}(\mathbb{Q} \text {-mod })_{+}\right)$, which we call $\phi^{*} N(X)$. This gives a functor $\phi^{*} N$ which is the right adjoint of a symmetric monoidal Quillen equivalence.

$$
L: S p^{\Sigma}\left(\mathrm{Ch}(\mathbb{Q}-\bmod )_{+}\right) \rightleftarrows S p^{\Sigma}(\mathrm{s} \mathbb{Q}-\bmod ): \phi^{*} N
$$

Let $C_{0}: \operatorname{Ch}(\mathbb{Q}$-mod $) \rightarrow \mathrm{Ch}(\mathbb{Q} \text {-mod })_{+}$be the functor which takes a chain complex $X$ to its [-1]-connective cover. Thus $C_{0} X_{n}$ is $X_{n}$ for $n>0$, zero for $n<0$ and $\operatorname{ker}\left(\partial: X_{0} \rightarrow X_{-1}\right)$ for $n=0$. For a chain complex $Y$, let $R Y$ be the symmetric spectrum with $(R Y)_{n}=C_{0}(Y \otimes \mathbb{Q}[n])$. This has a left adjoint $D$; thus we obtain the final adjoint pair which is a strong symmetric monoidal Quillen equivalence.

$$
D: S p^{\Sigma}\left(\mathrm{Ch}(\mathbb{Q} \text {-mod })_{+}\right) \rightleftarrows \mathrm{Ch}(\mathbb{Q} \text {-mod }): R
$$

We can give an explicit definition of $D$ taken from [Shi07a]. Let $I$ be the skeleton of the category of finite sets and injections with objects $\mathbf{n}$. For $X \in S p^{\Sigma}\left(\mathrm{Ch}(\mathbb{Q}-\bmod )_{+}\right)$, define a functor $D_{X}: I \rightarrow \operatorname{Ch}\left(\mathbb{Q}\right.$-mod) by $D_{X}(\mathbf{n})=\mathbb{Q}[-n] \otimes X_{n}$. There are structure maps $\sigma: \mathbb{Q}[m-n] \otimes X_{n} \rightarrow X_{m}$ with adjoints $\tilde{\sigma}: X_{n} \rightarrow \mathbb{Q}[n-m] \otimes X_{m}$. For a standard inclusion of a subset $\alpha: \mathbf{n} \rightarrow \mathbf{m}$, the map $D_{X}(\alpha)$ is $\operatorname{Id}_{\mathbb{Q}[-n]} \otimes \widetilde{\sigma}$. For an isomorphism in $\mathrm{I}$, the action is given by the tensor product of the action on $X_{n}$ and the sign action on $\mathbb{Q}[-n]$. The functor $D: S p^{\Sigma}\left(\mathrm{Ch}(\mathbb{Q} \text {-mod })_{+}\right) \rightarrow \mathrm{Ch}(\mathbb{Q}$-mod $)$ is defined by $D X=\operatorname{colim}_{I} D_{X}$.

There has been some confusion over whether or not $D$ is symmetric monoidal;

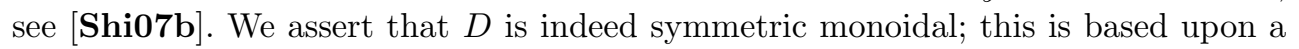
personal communication with Neil Strickland.

Now we can start constructing our new category $\mathcal{E}_{t}$. The category $\mathcal{E}_{\text {top }}^{H}$ is enriched over symmetric spectra and we have given $\bmod -\mathcal{E}_{\text {top }}^{H}$ a model structure using $S p_{+}^{\Sigma}$. Let us temporarily call this $\left(\bmod -\mathcal{E}_{t o p}^{H}\right)_{+}$. We can also consider the category mod- $\mathcal{E}_{t o p}^{H}$ with model structure defined via $S p^{\Sigma}$ (that is, using the stable model structure). Since the weak equivalences are the same in both cases and the fibrations are defined object-wise we have the following.

Lemma 6.2. The identity functor from $\left(\bmod -\mathcal{E}_{\text {top }}^{H}\right)_{+}$to $\bmod -\mathcal{E}_{\text {top }}^{H}$ is the left adjoint of a Quillen equivalence.

From here on, we only consider $\bmod -\mathcal{E}_{\text {top }}^{H}$ with the model structure induced from $S p^{\Sigma}$. This is a much better behaved category as now the unit is cofibrant.

Let $\widetilde{\mathbb{Q}} \mathcal{E}_{\text {top }}^{H}$ be the $S p^{\Sigma}\left(\mathrm{s} \mathbb{Q}\right.$-mod)-enriched category with object set $\mathcal{G}_{\text {top }}^{H}$ and morphisms defined by $\left(\widetilde{\mathbb{Q}} \mathcal{E}_{\text {top }}^{H}\right)(a, b)=\widetilde{\mathbb{Q}}\left(\mathcal{E}_{\text {top }}^{H}(a, b)\right)$. This construction is a simplification of $\left[\right.$ DS07, Proposition A.3b]. Since $\widetilde{\mathbb{Q}}$ is symmetric monoidal, $\widetilde{\mathbb{Q}} \mathcal{E}_{\text {top }}^{H}$ is a symmetric monoidal enriched category. We repeat this operation twice more to form 
$D \phi^{*} N \tilde{\mathbb{Q}} \mathcal{E}_{\text {top }}^{H}$, which is a symmetric monoidal $\mathrm{Ch}(\mathbb{Q}$-mod $)$-enriched category. We define $\mathcal{E}_{t}^{H}$ as $D \phi^{*} N \tilde{\mathbb{Q}} \mathcal{E}_{\text {top }}^{H}$.

Proposition 6.3. For each of the adjoint pairs $(\tilde{\mathbb{Q}}, U),\left(L, \phi^{*} N\right)$ and $(D, R)$, there is an induced Quillen equivalence as below:

$$
\begin{aligned}
\tilde{\mathbb{Q}}: \bmod -\mathcal{E}_{\text {top }}^{H} & \rightleftarrows \bmod -\tilde{\mathbb{Q}} \mathcal{E}_{t o p}^{H}: U^{\prime}, \\
L^{\prime}: \bmod -\phi^{*} N \tilde{\mathbb{Q}} \mathcal{E}_{t o p}^{H} & \rightleftarrows \bmod -\tilde{\mathbb{Q}} \mathcal{E}_{t o p}^{H}: \phi^{*} N, \\
D: \bmod -\phi^{*} N \tilde{\mathbb{Q}} \mathcal{E}_{t o p}^{H} & \rightleftarrows \bmod -D \phi^{*} N \tilde{\mathbb{Q}} \mathcal{E}_{t o p}^{H}: R^{\prime} .
\end{aligned}
$$

Proof. The induced adjunctions are defined in [SS03a, Section 3] and we give brief details below. Since $\tilde{\mathbb{Q}}$ and $D$ are strong monoidal these pass to the categories of modules as above without change. The right adjoint $\phi^{*} N$ also passes directly to the module categories, whereas all the other functors must be changed. The right adjoints $U^{\prime}$ and $R^{\prime}$ are slight alterations of $U$ and $R$, which we demonstrate for $U^{\prime}$. Take a $\tilde{\mathbb{Q}} \mathcal{E}_{\text {top }}^{H}$-module $M$, then $U^{\prime} M(\sigma)=U(M(\sigma))$. We must then give the maps

$$
U M(\sigma) \wedge \mathcal{E}_{\text {top }}^{H}\left(\sigma^{\prime}, \sigma\right) \rightarrow U M\left(\sigma^{\prime}\right) .
$$

We do so by applying the unit map $\mathcal{E}_{\text {top }}^{H}\left(\sigma^{\prime}, \sigma\right) \rightarrow U \tilde{\mathbb{Q}} \mathcal{E}_{\text {top }}^{H}\left(\sigma^{\prime}, \sigma\right)$ and then using the monoidality of $U$ and the action map of $M$. The left adjoint $L^{\prime}$ is more complicated, since it is not strong monoidal. Take a $\phi^{*} N \tilde{\mathbb{Q}} \mathcal{E}_{\text {top }}^{H}$-module $M$; then $L^{\prime} M(a)$ is defined as the coequaliser of the following diagram (we describe the maps below):

$$
\bigvee_{b, c} L\left(M(b) \wedge \phi^{*} N \tilde{\mathbb{Q}} \mathcal{E}_{\text {top }}^{H}(c, b)\right) \wedge \tilde{\mathbb{Q}} \mathcal{E}_{\text {top }}^{H}(a, c) \Longrightarrow \bigvee_{d} L M(d) \wedge \tilde{\mathbb{Q}} \mathcal{E}_{\text {top }}^{H}(a, d)
$$

One map is induced by the action of $\phi^{*} N \tilde{\mathbb{Q}} \mathcal{E}_{\text {top }}^{H}$ on $M$ and the other is the composite of the op-monoidal structure on $L$, the counit of $\left(L, \phi^{*} N\right)$ and the composition map of $\tilde{\mathbb{Q}} \mathcal{E}_{\text {top }}^{H}$.

The pair $\left(\tilde{\mathbb{Q}}, U^{\prime}\right)$ induce a Quillen pair between mod- $\mathcal{E}_{t o p}^{H}$ and mod- $\tilde{\mathbb{Q}} \mathcal{E}_{t o p}^{H}$. The free modules are a set of generators for these categories. Since for each $a$ and $b$ in $\mathcal{G}_{\text {top }}^{H}$, $\mathcal{E}_{\text {top }}^{H}(a, b)$ has rational homotopy groups, it follows that the unit and counit for the derived adjunctions are equivalences on these generators and thus $\left(\tilde{\mathbb{Q}}, U^{\prime}\right)$ is a Quillen equivalence. Since $\left(L, \phi^{*} N\right)$ and $(D, R)$ are Quillen equivalences, [SS03a, Theorem $6.5]$ implies that the other two pairs are Quillen equivalences.

Proposition 6.4. The Quillen equivalences $\left(\tilde{\mathbb{Q}}, U^{\prime}\right)$ and $\left(D, R^{\prime}\right)$ are strong symmetric monoidal Quillen equivalences. The adjunction $\left(L^{\prime}, \phi^{*} N\right)$ is a symmetric monoidal Quillen equivalence.

Proof. The first statement is a routine exercise in manipulating coends. The second requires some more work. It is easy to see that $\phi^{*} N$ induces a symmetric monoidal functor mod- $\tilde{\mathbb{Q}} \mathcal{E}_{t o p}^{H} \rightarrow \bmod -\phi^{*} N \tilde{\mathbb{Q}} \mathcal{E}_{t o p}^{H}$.

We must show that

$$
\eta: L^{\prime}\left(\phi^{*} N \tilde{\mathbb{Q}} \mathcal{E}_{\text {top }}^{H}\left(-, S_{H}\right)\right) \rightarrow \tilde{\mathbb{Q}} \mathcal{E}_{\text {top }}^{H}\left(-, S_{H}\right)
$$

is a weak equivalence and that for cofibrant $\phi^{*} N \tilde{\mathbb{Q}} \mathcal{E}_{t o p}^{H}$-modules $X$ and $Y$, the map 
$m: L^{\prime}(X \square Y) \rightarrow L^{\prime} X \square L^{\prime} Y$ (which exists since $\phi^{*} N$ is monoidal) is a weak equivalence. It suffices to prove the second condition for the free modules of mod- $\phi^{*} N \tilde{\mathbb{Q}} \mathcal{E}_{\text {top }}^{H}$, since these generate the homotopy category. Thus, to prove both statements, we only need consider the behaviour of $L^{\prime}$ on the free modules. Let $\operatorname{sym}(\mathbb{Q}[1])$ denote the unit of $S p^{\Sigma}\left(\mathrm{Ch}(\mathbb{Q}-\bmod )_{+}\right)$and $\operatorname{sym}\left(\widetilde{\mathbb{Q}} S^{1}\right)$ be the unit of $S p^{\Sigma}(\mathrm{s} \mathbb{Q}-\bmod )$, these are both cofibrant. Then

$$
L^{\prime}\left(\phi^{*} N \tilde{\mathbb{Q}} \mathcal{E}_{\text {top }}^{H}(-, a)\right) \cong\left(\tilde{\mathbb{Q}} \mathcal{E}_{\text {top }}^{H}(-, a)\right) \otimes L \operatorname{sym}(\mathbb{Q}[1]) .
$$

Recall the following three points:

- there is a natural isomorphism $F_{g} \square F_{k} \cong F_{g \wedge k}$,

- weak equivalences are defined levelwise in categories of right modules, and

- smashing with a cofibrant object of $S p^{\Sigma}(\mathrm{s} \mathbb{Q}$-mod) preserves weak equivalences (see the proof of [Shi07a, Corollary 3.4]).

Thus all we need prove is that

$$
\operatorname{Lymm}(\mathbb{Q}[1]) \rightarrow \operatorname{sym}\left(\widetilde{\mathbb{Q}} S^{1}\right)
$$

and

$$
\operatorname{Lsym}(\mathbb{Q}[1]) \rightarrow \operatorname{Lsym}(\mathbb{Q}[1]) \otimes L \operatorname{sym}(\mathbb{Q}[1])
$$

are weak equivalences. This a part of [Shi07a, Proposition 4.4] which states that $\left(L, \phi^{*} N\right)$ is a monoidal Quillen equivalence.

By [Shi07a, Proposition 4.4, Lemma 4.8 and the proof of Theorem 1.2], the functors $\phi^{*} N, D$ and $\widetilde{\mathbb{Q}}$ preserve all weak equivalences. Thus we do not need to consider derived functors in the following work.

Now we give a monoidal isomorphism of categories enriched over graded $\mathbb{Q}$-modules between $\mathrm{H}_{*} \mathcal{E}_{t}^{H}$ and $\pi_{*} \mathcal{E}_{\text {top }}^{H}$. Consider $\pi_{*} \mathcal{E}_{\text {top }}^{H}(a, b)$ which we define as $\left[S, \mathcal{E}_{\text {top }}^{H}(a, b)\right]_{*}^{\Sigma}$, graded maps in the homotopy category of symmetric spectra. We can apply the functor $\widetilde{\mathbb{Q}}$ to obtain a map as below, with the right-hand side the set of graded maps in the homotopy category of symmetric spectra in simplicial $\mathbb{Q}$-modules.

$$
\left[S, \mathcal{E}_{\text {top }}^{H}(a, b)\right]_{*}^{\Sigma} \rightarrow\left[\widetilde{\mathbb{Q}} S, \widetilde{\mathbb{Q}} \mathcal{E}_{\text {top }}^{H}(a, b)\right]_{*}^{\mathrm{s} \mathbb{Q}} .
$$

We call the right-hand side of the above $\pi_{*} \widetilde{\mathbb{Q}} \mathcal{E}_{\text {top }}^{H}(a, b)$. This is an isomorphism since $\mathcal{E}_{\text {top }}^{H}(a, b)$ has rational homotopy groups. Furthermore, this preserves the monoidal structures as we now explain. Taking the smash product of symmetric spectra we obtain the following maps:

$$
\begin{aligned}
{\left[S, \mathcal{E}_{\text {top }}^{H}(a, b)\right]_{*}^{\Sigma} \otimes\left[S, \mathcal{E}_{\text {top }}^{H}(c, d)\right]_{*}^{\Sigma} } & \longrightarrow\left[S, \mathcal{E}_{\text {top }}^{H}(a, b) \wedge \mathcal{E}_{\text {top }}^{H}(c, d)\right]_{*}^{\Sigma} \\
& \longrightarrow\left[S, \mathcal{E}_{\text {top }}^{H}(a \wedge c, b \wedge d)\right]_{*}^{\Sigma} .
\end{aligned}
$$

This defines the monoidal structure on $\pi_{*} \mathcal{E}_{\text {top }}^{H}$. One defines a monoidal structure on $\pi_{*} \widetilde{\mathbb{Q}} \mathcal{E}_{\text {top }}^{H}$ similarly. We can apply $\widetilde{\mathbb{Q}}$ to the various stages of the above and obtain a large commuting diagram which implies that $\widetilde{\mathbb{Q}}: \pi_{*} \mathcal{E}_{\text {top }}^{H} \rightarrow \pi_{*} \widetilde{\mathbb{Q}} \mathcal{E}_{\text {top }}^{H}$ is an isomorphism of symmetric monoidal enriched categories. We repeat this using $\phi^{*} N$ and $D$ noting that $D \phi^{*} N \widetilde{\mathbb{Q}} S$ is weakly equivalent to $\mathbb{Q}$ and that for any chain complex $X$, $\mathrm{H}_{*} X \cong[\mathbb{Q}, X]_{*}^{\mathrm{Ch}}$ graded maps in the homotopy category of rational chain complexes. 
Thus we have obtained an isomorphism of symmetric monoidal enriched categories $\pi_{*} \mathcal{E}_{t o p}^{H} \rightarrow \mathrm{H}_{*} D \phi^{*} N \widetilde{\mathbb{Q}} \mathcal{E}_{\text {top }}^{H}$. This section is summarised in the following theorem; see also [GS, Theorem 4.1].

Theorem 6.5. Let $\mathcal{E}_{t}^{H}$ be the symmetric monoidal $\mathrm{Ch}(\mathbb{Q}$-mod)-enriched category $D \phi^{*} N \tilde{\mathbb{Q}} \mathcal{E}_{\text {top }}^{H}$. There is a zig-zag of monoidal Quillen equivalences between mod-E $\mathcal{E}_{\text {top }}^{H}$ (enriched over $S p_{+}^{\Sigma}$ ) and a category $\bmod -\mathcal{E}_{t}^{H}$ (which is enriched over $\mathrm{Ch}(\mathbb{Q}$-mod)). This zig-zag induces an isomorphism of monoidal graded $\mathbb{Q}$-categories:

$$
\pi_{*}\left(\mathcal{E}_{\text {top }}^{H}\right) \rightarrow \mathrm{H}_{*} \mathcal{E}_{t}^{H} .
$$

Remark 6.6. We consider the above theorem in the case of the trivial group where our work reduces to that of [Shi07a]. Write $S_{\mathbb{Q}}$ for $S_{\{e\}}$. Here $\mathcal{G}_{\text {top }}$ has just one object and mod- $\mathcal{E}_{\text {top }}$ is equivalent to $S_{\mathbb{Q}}$-mod. Moving from mod- $\mathcal{E}_{\text {top }}$ to mod- $\mathcal{E}_{t}$ is then just applying the functors of [Shi07a] to the spectrum $S_{\mathbb{Q}}$. The resulting chain complex is then weakly equivalent to $\mathbb{Q}$, as the comparison between mod- $\mathcal{E}_{t}$ and $\bmod -\mathcal{E}_{a}$ below will prove.

We now have two $\mathrm{Ch}\left(\mathbb{Q}\right.$-mod)-enriched categories, $\mathcal{E}_{t}^{H}$ and $\mathcal{E}_{a}^{H}$. While the definition of $\mathcal{E}_{t}^{H}$ is somewhat complicated, we have control over its homology.

\section{Comparing $\mathcal{E}_{t}^{H}$ and $\mathcal{E}_{a}^{H}$}

We show that the homology of $\mathcal{E}_{t}^{H}$ is isomorphic, as an enriched category, to $\mathcal{E}_{a}^{H}$ in Theorem 7.4. Furthermore, this isomorphism respects the monoidal structures. Theorem 7.5 implies that $\mathcal{E}_{t}^{H}$ and $\mathrm{H}_{*} \mathcal{E}_{t}^{H}$ are 'quasi-isomorphic'; thus by Corollaries 7.6 and 7.7, $\bmod -\mathcal{E}_{t}^{H}, \bmod -\mathrm{H}_{*} \mathcal{E}_{t}^{H}$ and $\bmod -\mathcal{E}_{a}^{H}$ are Quillen equivalent by strong symmetric monoidal adjunctions.

This completes the main part of this paper, as we have now shown that our algebraic model is Quillen equivalent to the category of rational $G$-spectra. We leave the consideration of algebras and modules over an algebra to the last section.

We give a summary of [May96, Chapter XIX, Theorem 5.6] below, and we will use this result in our calculations. Recall that for a $G$-spectrum $X, \pi_{*}^{H}(X) \cong\left[G / H_{+}, X\right]_{*}^{G}$. The right-hand side admits an action of the Weyl group $W_{G} H=N_{G} H / H$ since there is a $G$-map $G / H \times W_{G} H \rightarrow G / H$ given by $(g H, n H) \mapsto g n H$. Hence, $\pi_{*}^{H}(X) \otimes \mathbb{Q}$ is a $\mathbb{Q} W_{G} H$-module. Recall the idempotent of the rational Burnside ring $e_{H}$ from Section 5 ; then we have a $\mathbb{Q} W_{G} H$-module $\iota_{H}^{*}\left(e_{H}\right)_{*} \pi_{*}^{H}(X) \otimes \mathbb{Q}$. For the rest of this section we will write $e_{H}$ for $\iota_{H}^{*}\left(e_{H}\right)_{*}$.

Theorem 7.1. For $G$-spectra $X$ and $Y$, there is an isomorphism of graded rational vector spaces

$$
[X, Y]_{\mathbb{Q}}^{G} \cong \bigoplus_{(H) \leqslant G} \operatorname{Hom}_{\mathbb{Q}}\left(e_{H} \pi_{*}^{H}(X) \otimes \mathbb{Q}, e_{H} \pi_{*}^{H}(X) \otimes \mathbb{Q}\right)^{W_{G} H} .
$$

We can relate the above result to our work via the following isomorphisms. Recall the space $E\langle H\rangle$, which is the cofibre of the map $E[<H]_{+} \rightarrow E[\leqslant H]_{+}$and is rationally equivalent to $e_{H} S$. We write $[X, Y]_{\mathbb{Q}}^{G \mid H}$ for maps in the homotopy category of $L_{E\langle H\rangle} G \mathcal{M}_{\mathbb{Q}}$ and $\widehat{f}_{H}$ for fibrant replacement in this model category. The $G$-spectra 
$\widehat{f}_{H} Y$ and $e_{H} Y$ are rationally equivalent, since $e_{H} Y$ is $E\langle H\rangle$-local, which gives us the third isomorphism below.

$$
[X, Y]_{\mathbb{Q}}^{G} \cong \bigoplus_{(H) \leqslant G}[X, Y]_{\mathbb{Q}}^{G \mid H} \cong \bigoplus_{(H) \leqslant G}\left[X, \widehat{f}_{H} Y\right]_{\mathbb{Q}}^{G} \cong \bigoplus_{(H) \leqslant G}\left[X, e_{H} Y\right]_{\mathbb{Q}}^{G}
$$

If $X$ and $Y$ are $S_{H}$-modules, then $\pi_{*}^{H}(X) \cong e_{H} \pi_{*}^{H}(X) \otimes \mathbb{Q}$ and $[X, Y]_{\mathbb{Q}}^{G} \cong[X, Y]_{*}^{S_{H}}$, since every $S_{H}$-module is $S_{\mathcal{M}}^{0} \mathbb{Q} \wedge E\langle H\rangle$-local (Lemma 5.10). Thus, for $S_{H}$-modules $X$ and $Y$, we have an isomorphism of graded rational vector spaces

$$
[X, Y]_{*}^{S_{H}} \cong \operatorname{Hom}_{\mathbb{Q}}\left(\pi_{*}^{H}(X), \pi_{*}^{H}(Y)\right)^{W_{G} H} .
$$

Lemma 7.2. For $g H \in W_{G} H$ we have a map $* \rightarrow G / H$ which sends the point to $g H$. Using the unit map of $S_{H}$, this induces a map of $G$-spectra $\widetilde{g H}: S^{0} \rightarrow\left(\widehat{c} G / H_{+}\right) \wedge S_{H}$. The assignment $g H \mapsto \widetilde{g H}$ induces an isomorphism

$$
\mathbb{Q} W_{G} H \rightarrow \pi_{*}^{H}\left(\left(\widehat{c} G / H_{+}\right) \wedge S_{H}\right) .
$$

Proof. By the proof of Lemma 5.10, we can replace $S_{H}$ by $E\langle H\rangle \wedge S_{\mathcal{M}}^{0} \mathbb{Q}$, so the above is isomorphic to $\pi_{*}\left(\left(\left(\widehat{c} G / H_{+}\right) \wedge E\langle H\rangle \wedge S_{\mathcal{M}}^{0} \mathbb{Q}\right)^{H}\right)$. Let $\mathscr{F}_{H}$ be the family of proper subgroups of $H$, then we have a cofibre sequence of $H$-spaces $\left(E \mathscr{F}_{H}\right)_{+} \rightarrow S^{0} \rightarrow \widetilde{E} \mathscr{F}_{H}$. Now $E\left[\leqslant_{G} H\right]$ is $H$-equivariantly weakly equivalent to $S$, so $E\langle H\rangle$ is $H$-equivariantly weakly equivalent to $\widetilde{E} \mathscr{F}_{H}$. Thus we have an isomorphism (in fact one can define $\Phi^{H}$ as $\left.\left(\widetilde{E} \mathscr{F}_{H} \wedge(-)\right)^{H}\right)$

$$
\pi_{*}\left(\left(\widehat{c} G / H_{+} \wedge E\langle H\rangle \wedge S_{\mathcal{M}}^{0} \mathbb{Q}\right)^{H}\right) \cong \pi_{*}\left(\Phi^{H}\left(\widehat{c} G / H_{+} \wedge S_{\mathcal{M}}^{0} \mathbb{Q}\right)\right) .
$$

Since $\Phi^{H}$ commutes with smash products of cofibrant objects, cofibre sequences and coproducts (such as those used to define $S_{\mathcal{M}}^{0} \mathbb{Q}$ ) it follows that the above is isomorphic to $\pi_{*}\left(\Phi^{H}\left(\widehat{c} G / H_{+}\right)\right) \otimes \mathbb{Q}$. The following is standard: $\Phi^{H}\left(\widehat{c} G / H_{+}\right) \simeq \Sigma^{\infty}\left(G / H^{H}\right)=$ $\Sigma^{\infty} W_{G} H$, the suspension spectrum of a finite set. We have proved that the groups $\pi_{*}\left(\Phi^{H}\left(\widehat{c} G / H_{+}\right)\right) \otimes \mathbb{Q}$ and $\mathbb{Q} W_{G} H$ are isomorphic; thus, the groups $\pi_{*}^{H}\left(\left(\widehat{c} G / H_{+}\right) \wedge\right.$ $\left.S_{H}\right)$ and $\mathbb{Q} W_{G} H$ are isomorphic. It follows that the map specified in the lemma is a particular choice of isomorphism.

The same method as above proves that the maps below are isomorphisms for $i \geqslant 0$. The second map is induced by the smash product of $S_{H}$-modules.

$$
\left(\mathbb{Q} W_{G} H\right)^{\otimes_{i}} \rightarrow\left(\pi_{*}^{H}\left(\widehat{c} G / H_{+} \wedge S_{H}\right)\right)^{\otimes_{i}} \rightarrow \pi_{*}^{H}\left(\left(\widehat{c} G / H_{+}\right)^{\wedge_{i}} \wedge S_{H}\right) .
$$

It follows that for every $i, j \geqslant 0$, we have an isomorphism $\alpha=\alpha_{i, j}$ induced by the smash product:

$$
\pi_{*}^{H}\left(\left(\widehat{c} G / H_{+}\right)^{\wedge_{i}} \wedge S_{H}\right) \otimes \pi_{*}^{H}\left(\left(\widehat{c} G / H_{+}\right)^{\wedge_{j}} \wedge S_{H}\right) \rightarrow \pi_{*}^{H}\left(\left(\widehat{c} G / H_{+}\right)^{\wedge_{i+j}} \wedge S_{H}\right) .
$$

Recall the gr. $\left(\mathbb{Q}\right.$-mod)-enriched category $\pi_{*} \mathcal{E}_{\text {top }}^{H}$, for $a$ and $b$ in $\mathcal{G}_{\text {top }}^{H}$,

$$
\pi_{*} \mathcal{E}_{\text {top }}^{H}(a, b)=\pi_{*}(\underline{\operatorname{Hom}}(a, b)) .
$$

This category is symmetric monoidally isomorphic to $\mathrm{H}_{*} \mathcal{E}_{t}^{H}$ (Theorem 6.5). We also have the gr. $\left(\mathbb{Q}\right.$-mod)-enriched category on object set $\mathcal{G}_{\text {top }}^{H}$ with the morphism object defined by $[a, b]_{*}^{S_{H}}$, graded maps in homotopy category of $S_{H}$-modules (these morphism objects are rational since $S_{H}$ is fibrant in $\left.G \mathcal{M}_{\mathbb{Q}}\right)$. This symmetric monoidal 
enriched category, which we call Ho $\mathcal{E}_{\text {top }}^{H}$, is isomorphic to $\pi_{*} \mathcal{E}_{\text {top }}^{H}$ via the adjunctions of Section 5. Furthermore, since these adjunctions are symmetric monoidal, so is this isomorphism. For the purposes of calculations, it is easiest to work with objects of the form $[a, b]_{*}^{S_{H}}$.

Proposition 7.3. There is an isomorphism of symmetric monoidal gr. $(\mathbb{Q}$-mod)-enriched categories between $\mathrm{Ho}_{\text {top }}^{H}$ and the full gr. $(\mathbb{Q}$-mod)-enriched category on the objects $\pi_{*}^{H}(a) \in \operatorname{gr}$. $\left(\mathbb{Q} W_{G} H\right.$-mod $)$, for $a \in \mathcal{G}_{\text {top }}^{H}$. We denote this category by $\pi_{*} \mathcal{G}_{\text {top }}^{H}$.

Proof. Thus for $a$ and $b$ in $\mathcal{G}_{\text {top }}^{H}$, we have $\pi_{*} \mathcal{G}_{\text {top }}^{H}(a, b)=\operatorname{Hom}_{\mathbb{Q}}\left(\pi_{*}^{H}(a), \pi_{*}^{H}(b)\right)^{W_{G} H}$. Note that each $\pi_{*}^{H}(a)=[S, a]_{*}^{H}$ is concentrated in degree zero. The forgetful functor induces a map $[a, b]^{S_{H}} \rightarrow[a, b]^{H}$. Combining this with composition gives a map of graded $\mathbb{Q} W_{G} H$-modules $[a, b]_{*}^{S_{H}} \otimes_{\mathbb{Q}}[S, a]_{*}^{H} \rightarrow[S, b]_{*}^{H}$. This map has an adjoint, which is a map of graded $\mathbb{Q}$-modules $[a, b]_{*}^{S_{H}} \rightarrow \operatorname{Hom}_{\mathbb{Q}}\left([S, a]_{*}^{H},[S, b]_{*}^{H}\right)^{W_{G} H}$. Thus we have a gr. $(\mathbb{Q}$-mod)-enriched functor

$$
\pi_{*}^{H}(-)=[S,-]_{*}^{H}: \text { Ho } \mathcal{E}_{\text {top }}^{H} \rightarrow \pi_{*} \mathcal{G}_{\text {top }}^{H} .
$$

This functor is an isomorphism of gr. $(\mathbb{Q}$-mod)-enriched categories, since (as proven above) $[X, Y]_{*}^{S_{H}}$ and $\operatorname{Hom}_{\mathbb{Q}}\left(\pi_{*}^{H}(X), \pi_{*}^{H}(Y)\right)^{W_{G} H}$ are isomorphic. We now consider the diagram below, where everything is concentrated in degree zero:

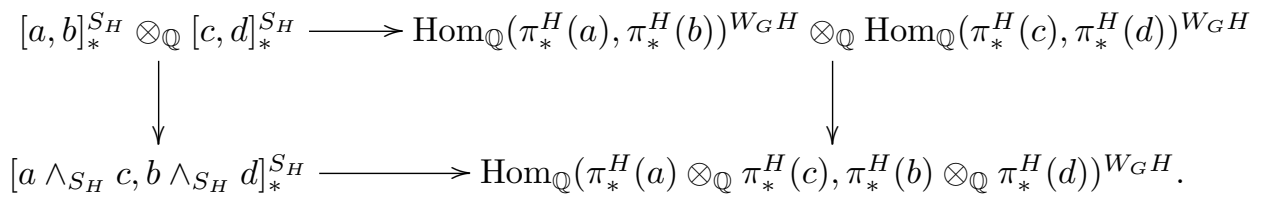

The lower horizontal map uses the isomorphism $\alpha$ (constructed above) from

$$
\pi_{*}^{H}(a) \otimes_{\mathbb{Q}} \pi_{*}^{H}(c) \quad \text { to } \quad \pi_{*}^{H}\left(a \wedge_{S_{H}} c\right) .
$$

This diagram commutes since for maps $f$ and $g$ the smash product of $\pi_{*}^{H}(f)$ and $\pi_{*}^{H}(g)$ is $\pi_{*}^{H}(f \wedge g)$.

Theorem 7.4. There is a symmetric monoidal isomorphism of gr.(QQQ-mod)-enriched categories $\mathcal{E}_{a}^{H} \rightarrow \mathrm{H}_{*} \mathcal{E}_{t}^{H}$.

Proof. We replace $\mathrm{H}_{*} \mathcal{E}_{t}^{H}$ by $\pi_{*} \mathcal{E}_{\text {top }}^{H}$, which is isomorphic to Ho $\mathcal{E}_{\text {top }}^{H}$ and hence to $\pi_{*} \mathcal{G}_{\text {top }}^{H}$.

The category $\pi_{*} \mathcal{G}_{\text {top }}^{H}$ has object set given by all smash products of $\left(\widehat{c} G / H_{+}\right) \wedge S_{H}$ (here we mean the smash product of $S_{H}$-modules), with $S_{H}$ as the zero fold smash product. Similarly, $\mathcal{E}_{a}^{H}$ has object set given by all tensor products of $\mathbb{Q} W_{G} H$ with $\mathbb{Q}$ as the zero fold tensor product. Define an isomorphism on these object sets by sending $\mathbb{Q} W_{G} H^{\otimes_{i}}$ to $\left(\widehat{c} G / H_{+}\right)^{\wedge_{i}} \wedge S_{H}$ for $i \geqslant 0$. In our work above, we have the specified isomorphisms

$$
\left(\mathbb{Q} W_{G} H\right)^{\otimes_{i}} \rightarrow\left(\pi_{*}^{H}\left(\left(\widehat{c} G / H_{+}\right) \wedge S_{H}\right)\right)^{\otimes_{i}} \rightarrow \pi_{*}^{H}\left(\left(\widehat{c} G / H_{+}\right)^{\wedge_{i}} \wedge S_{H}\right) .
$$

These maps induce an isomorphism between

$$
\operatorname{Hom}_{\mathbb{Q}}\left(\left(\mathbb{Q} W_{G} H\right)^{\otimes_{i}},\left(\mathbb{Q} W_{G} H\right)^{\otimes_{j}}\right)^{W_{G} H}
$$


and

$$
\operatorname{Hom}_{\mathbb{Q}}\left(\pi_{*}^{H}\left(\left(\widehat{c} G / H_{+}\right)^{\wedge_{i}} \wedge S_{H}\right), \pi_{*}^{H}\left(\left(\widehat{c} G / H_{+}\right)^{\wedge_{j}} \wedge S_{H}\right)\right)^{W_{G} H} .
$$

Thus we have an isomorphism of gr. $\left(\mathbb{Q}\right.$-mod)-enriched categories $\mathcal{E}_{a}^{H} \rightarrow \pi_{*} \mathcal{G}_{\text {top }}^{H}$. Each of these isomorphisms of gr.( $\mathbb{Q}$-mod)-enriched categories is symmetric monoidal, so the result holds.

We now know that $\mathrm{H}_{*} \mathcal{E}_{t}^{H}$ is concentrated in degree zero. Our next result uses this to provide a comparison between $\mathrm{H}_{*} \mathcal{E}_{t}^{H}$ and $\mathcal{E}_{t}^{H}$. Let $\mathcal{E}, \mathcal{D}$ be categories enriched over a model category, a map of enriched categories $\psi: \mathcal{E} \rightarrow \mathcal{D}$ is a quasi-isomorphism if it induces an isomorphism on the object sets and $\psi: \mathcal{E}(A, B) \rightarrow \mathcal{D}(\psi A, \psi B)$ is a weak equivalence for all pairs of objects $A$ and $B$.

Theorem 7.5. If $\mathcal{E}$ is a $\mathrm{Ch}\left(\mathbb{Q}\right.$-mod)-category with $\mathrm{H}_{*} \mathcal{E}$ concentrated in degree zero, then $\mathcal{E}$ is quasi-isomorphic to $\mathrm{H}_{*} \mathcal{E}$ as $\mathrm{Ch}(\mathbb{Q}$-mod)-categories. An explicit zig-zag is constructed below. If $\mathcal{E}$ is a symmetric monoidal enriched category then the zig-zag consists of maps of symmetric monoidal enriched categories.

Proof. We will create a $\mathrm{Ch}\left(\mathbb{Q}\right.$-mod)-enriched category $C_{0} \mathcal{E}$ and a zig-zag of quasiisomorphisms: $\mathcal{E} \stackrel{\sim}{\sim} C_{0} \mathcal{E} \stackrel{\sim}{\longrightarrow} \mathrm{H}_{0} \mathcal{E}=\mathrm{H}_{*} \mathcal{E}$.

As in Section 6, we will use a symmetric monoidal adjunction to construct $C_{0} \mathcal{E}$. We have the $(-1)$-connective cover functor $C_{0}$, which is the right adjoint to the inclusion of $\mathrm{Ch}(\mathbb{Q} \text {-mod })_{+}$into $\mathrm{Ch}(\mathbb{Q}$-mod $)$. So for a chain complex $X,\left(C_{0} X\right)_{n}$ is $X_{n}$ for $n>0$, zero for $n<0$ and is given by $\operatorname{ker}\left(\partial: X_{0} \rightarrow X_{-1}\right)$ for $n=0$. This adjunction is strong symmetric monoidal and furthermore the counit is a symmetric monoidal natural transformation. Define $C_{0} \mathcal{E}$ to have the same set of objects as $\mathcal{E}$ and let $\left(C_{0} \mathcal{E}\right)(a, b)=C_{0}(\mathcal{E}(a, b))$. This is a symmetric monoidal $\mathrm{Ch}(\mathbb{Q}$-mod $)$-enriched category. The counit gives a map of symmetric monoidal $\mathrm{Ch}(\mathbb{Q}$-mod)-enriched categories $C_{0} \mathcal{E} \rightarrow \mathcal{E}$. This follows from the commutative diagram below, where $X \otimes Y \rightarrow Z$ is a map in $\mathrm{Ch}(\mathbb{Q}$-mod)

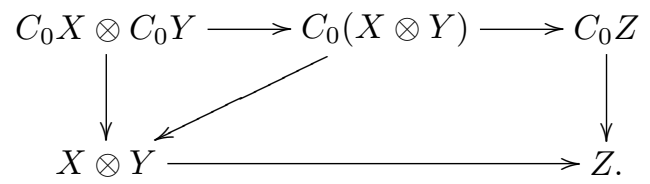

For a chain complex of $\mathbb{Q}$-modules, $X$, we have a map $C_{0} X \rightarrow \mathrm{H}_{0} X$ which sends $X_{i}$ to zero for $i>0$ and sends $\left(C_{0}(X)\right)_{0}=\operatorname{ker}\left(\partial_{0}\right) \rightarrow \mathrm{H}_{0} X$ by the quotient. We can consider $\mathrm{H}_{0}$ as a functor $\mathrm{Ch}(\mathbb{Q} \text {-mod })_{+} \rightarrow \mathbb{Q}$-mod, this has a right adjoint which includes $\mathbb{Q}$-mod into $\mathrm{Ch}(\mathbb{Q}$-mod) + by taking a $\mathbb{Q}$-module $M$ to the chain complex with $M$ in degree zero and zeroes elsewhere. The map $C_{0} X \rightarrow \mathrm{H}_{0} X$ is induced by the unit of this adjunction. The functor $\mathrm{H}_{0}$ is monoidal, as is the inclusion of $\mathbb{Q}$-mod into $\mathrm{Ch}(\mathbb{Q} \text {-mod })_{+}$. Thus we obtain a symmetric monoidal $\mathrm{Ch}(\mathbb{Q}$-mod $)$-category $\mathrm{H}_{0} \mathcal{E}$. Furthermore, the map $C_{0} X \rightarrow \mathrm{H}_{0} X$ is induced by the unit of the adjunction which is a symmetric monoidal natural transformation. As above, we obtain a map of symmetric monoidal $\mathrm{Ch}\left(\mathbb{Q}\right.$-mod)-enriched categories $C_{0} \mathcal{E} \rightarrow \mathrm{H}_{0} \mathcal{E}=\mathrm{H}_{*} \mathcal{E}$, which is a quasiisomorphism. 
For a map of $\mathrm{Ch}(\mathbb{Q}$-mod)-enriched categories $\psi: \mathcal{E} \rightarrow \mathcal{D}$, there is an adjoint pair of extension and restriction of scalars as defined in [SS03b, Section A.1]

$$
-\otimes_{\mathcal{E}} \mathcal{D}: \bmod -\mathcal{E} \rightleftarrows \bmod -\mathcal{D}: \psi^{*} .
$$

The left adjoint is given in terms of a coend:

$$
\left(M \otimes_{\mathcal{E}} \mathcal{D}\right)(a)=\int^{a} M(a) \otimes \mathcal{D}(-, \psi(a)) .
$$

The right adjoint simply lets $\mathcal{E}$ act on a $\mathcal{D}$-module via $\psi$. These form a Quillen pair that is a Quillen equivalence if $\psi$ is a quasi-isomorphism by [SSO3b, Theorem A.1.1]. If the map $\psi$ is symmetric monoidal, then it is routine to prove that $\left(-\otimes_{\mathcal{E}} \mathcal{D}, \psi^{*}\right)$ is a strong symmetric monoidal Quillen pair.

Corollary 7.6. For each subgroup H, Theorem 7.5 specifies a zig-zag of symmetric monoidal quasi-isomorphisms of $\mathrm{Ch}(\mathbb{Q}$-mod)-categories

$$
\mathcal{E}_{t}^{H} \stackrel{\sim}{\sim} C_{0} \mathcal{E}_{t}^{H} \stackrel{\sim}{\longrightarrow} \mathrm{H}_{*} \mathcal{E}_{t}^{H}
$$

hence there is an induced zig-zag of symmetric monoidal Quillen equivalences of $\mathrm{Ch}(\mathbb{Q}$-mod)-model categories

$$
\bmod -\mathcal{E}_{t}^{H} \leftrightarrows \bmod -C_{0} \mathcal{E}_{t}^{H} \rightleftarrows \bmod -\mathrm{H}_{*} \mathcal{E}_{t}^{H}
$$

We note that the above theorem and corollary are similar to results in [Shi02, Section 5]. Now we prove that the monoidal structures of $\mathcal{E}_{a}^{H}$ and $\mathrm{H}_{*} \mathcal{E}_{t}^{H}$ are equivalent; thus we can complete our symmetric monoidal comparison between mod- $\mathcal{E}_{a}^{H}$ and $\bmod -\mathcal{E}_{t}^{H}$.

Corollary 7.7. The Quillen pair of extension and restriction of scalars gives a strong symmetric monoidal Quillen equivalence (and an equivalence of categories) between $\mathcal{E}_{a}^{H}$-mod and $\mathrm{H}_{*} \mathcal{E}_{t}^{H}$-mod.

\section{Main results}

Theorem 8.1. For $G$ a finite group, there is a zig-zag of symmetric monoidal Quillen equivalences between the category of rational $G$-equivariant spectra and the algebraic model: $\prod_{(H) \leqslant G} \mathrm{Ch}\left(\mathbb{Q} W_{G} H\right.$-mod $)$.

Proof. We begin with Corollary 5.7, which splits rational $G$-spectra into the product $\prod_{(H) \leqslant G} L_{E\langle H\rangle} G \mathcal{M}_{\mathbb{Q}}$. Applying Proposition 5.11 to each factor of this category allows us to move to $\prod_{(H) \leqslant G} S_{H}$-mod. Next we use Theorem 5.14 to move to modules over a $S p^{\Sigma}$-enriched category, $\prod_{(H) \leqslant G} \bmod -\mathcal{E}_{t o p}^{H}$. We move to algebra, $\prod_{(H) \leqslant G} \bmod -\mathcal{E}_{t}^{H}$, with Theorem 6.5, and then use Corollaries 7.6 and 7.7 to get to the category $\prod_{(H) \leqslant G} \bmod -\mathcal{E}_{a}^{H}$. Finally we use Theorem 4.9 to complete the result.

Since we have symmetric monoidal functors we can apply [SS03a, Theorem 3.12] to each stage of the comparison to obtain the following corollaries. The unit of $S_{H}$-mod is not cofibrant, but this presents no difficulty. 
Corollary 8.2. For each subgroup $H$, the above zig-zag induces a zig-zag of Quillen equivalences between the category of algebras in $S_{H}$-mod and the category of algebras in $\mathrm{Ch}\left(\mathbb{Q} W_{G} H\right.$-mod).

Let $i: C_{0} \mathcal{E}_{t}^{H} \rightarrow \mathcal{E}_{t}^{H}$ and $p: C_{0} \mathcal{E}_{t}^{H} \rightarrow \mathrm{H}_{*} \mathcal{E}_{t}^{H}$ be the maps constructed in Corollary 7.6. Let $\psi: \mathcal{E}_{a}^{H} \rightarrow \mathrm{H}_{*} \mathcal{E}_{t}^{H}$ be the isomorphism constructed in Theorem 7.4 , since this is an isomorphism we can write $\left(\psi^{-1}\right)^{*}$ for the left adjoint to $\psi^{*}$. The composites $D \circ \phi^{*} N \circ \widetilde{\mathbb{Q}}$ and $U^{\prime} \circ L^{\prime} \widehat{c} \circ R^{\prime}$ give a derived equivalence between $\operatorname{Ho}\left(\mathcal{E}_{\text {top }}^{H}\right.$-mod $)$ and $\mathrm{Ho}\left(\mathcal{E}_{t}^{H}\right.$-mod $)$ as stated in Theorem 6.5. Note that no cofibrant replacements $(\widehat{c})$ are needed in the first of these composites as we are working rationally. The functor $L^{\prime}$ is the alteration of $L$ to modules over an enriched category as we have described in Section 6 . We will then need to alter $L^{\prime}$ so that it acts on categories of algebras, using the notation of [SS03a] we define $L^{\prime \prime}=\left(L^{\prime}\right)^{\text {mon }}$. We write out the derived composite functors needed for the next corollary. That such functors exist is perhaps of more interest than the explicit formulas; see also Section 3. The terms Id $\widehat{c}$ and Id $\widehat{f}$ are from the adjunction of Lemma 6.2 , where we change from the $S p_{+}^{\Sigma}$ to $S p^{\Sigma}$.

Definition 8.3. Let $\Theta$ be the derived functor

$$
(-) \otimes_{\mathcal{E}_{a}^{H}} \mathcal{G}_{a}^{H} \circ \psi^{*} \circ(\widehat{c}-) \otimes_{C_{0} \mathcal{E}_{t}^{H}} \mathrm{H}_{*} \mathcal{E}_{t}^{H} \circ i^{*} \circ D \circ \phi^{*} N \circ \widetilde{\mathbb{Q}} \circ \operatorname{Id} \widehat{c} \circ \underline{\operatorname{Hom}}\left(\mathcal{G}_{\text {top }}^{H},-\right)
$$

from $S_{H-\bmod }$ to $\mathbb{Q} W_{G} H$-mod. Let $\mathbb{H}$ be the derived functor

$$
(\widehat{c}-) \wedge_{\mathcal{E}_{\text {top }}^{H}} \mathcal{G}_{\text {top }}^{H} \circ \operatorname{Id} \widehat{f} \circ U^{\prime} \circ L^{\prime \prime} \widehat{c} \circ R^{\prime} \circ(\widehat{c}-) \otimes_{C_{0} \mathcal{E}_{t}^{H}} \mathcal{E}_{t}^{H} \circ p^{*} \circ\left(\psi^{-1}\right)^{*} \circ \underline{\operatorname{Hom}}\left(\mathcal{G}_{a}^{H},-\right)
$$

from $\mathbb{Q} W_{G} H$-mod to $S_{H}$-mod.

Corollary 8.4. For each $S_{H}$-algebra $A$ there is a zig-zag of Quillen equivalences between $A$-mod and $\Theta A$-mod. For each $\mathbb{Q} W_{G} H$-algebra $B$ there is a zig-zag of Quillen equivalences between $B$-mod and $\mathbb{H} B$-mod.

Remark 8.5. We note here that we have made no statement regarding model categories of commutative algebras. This is because not all of the model categories that we use have been shown to have model categories of commutative algebras. We expect that if this technical problem is solved, then our result will imply that the model categories of commutative algebras in $G \mathcal{M}_{\mathbb{Q}}$ and commutative algebras in the algebraic model are Quillen equivalent.

In particular, one would have to construct a model category of commutative algebras in $\bmod -\mathcal{E}_{\text {top }}^{H}$, when we are using the model structure arising from $S p^{\Sigma}$ (see Lemma 6.2). Recall that the usual model structure of commutative algebras in $S p^{\Sigma}$ is constructed from the positive model structure, $S p_{+}^{\Sigma}$.

\section{References}

[Ada74] J.F. Adams, Stable homotopy and generalised homology, Chicago Lectures in Math. University of Chicago Press, Chicago, Ill., 1974.

[Bar08] D. Barnes, Rational equivariant spectra, Ph.D. thesis, University of Sheffield, 2008, arXiv: 0802:0954v1[math.AT]. 
[Bor94] F. Borceux, Handbook of categorical algebra. 2, Categories and structures, Encyclopedia of Mathematics and its Applications 51, Cambridge University Press, Cambridge, 1994.

[Day70] B. Day, On closed categories of functors, in Reports of the Midwest category seminar, IV, Lecture Notes in Math. 137, 1-38, SpringerVerlag, New York, 1970.

[DS07] D. Dugger and B. Shipley, Enriched model categories and an application to additive endomorphism spectra, Theory Appl. Categ. 18 (2007), 400-439 (electronic).

[EKMM97] A.D. Elmendorf, I. Kriz, M.A. Mandell, and J.P. May, Rings, modules, and algebras in stable homotopy theory, Mathematical Surveys and Monographs 47, Amer. Math. Soc., Providence, RI, 1997. With an appendix by M. Cole.

[GM95] J.P.C. Greenlees and J.P. May, Generalized Tate cohomology, Mem. Amer. Math. Soc. 543 (1995), viii+178.

[GS] J.P.C. Greenlees and B. Shipley, An algebraic model for rational torusequivariant spectra, preprint, available on the Internet at http:// wWw.greenlees.staff.shef.ac.uk/preprints/tnq3.dvi.

[Hov99] M Hovey, Model categories, Mathematical Surveys and Monographs 63, Amer. Math. Soc., Providence, RI, 1999.

[Hov01] M Hovey, Spectra and symmetric spectra in general model categories, J. Pure Appl. Algebra 165 (2001), no. 1, 63-127.

[Kel05] G.M. Kelly, Basic concepts of enriched category theory, Repr. Theory Appl. Categ. 10, vi+137 pp. (electronic), 2005. Reprint of the 1982 original [Cambridge Univ. Press, Cambridge; MR0651714].

[LMSM86] L.G. Lewis, Jr., J.P. May, M. Steinberger, and J.E. McClure, Equivariant stable homotopy theory, Lecture Notes in Math. 1213, SpringerVerlag, New York, 1986. With contributions by J.E. McClure.

[Mac71] S. MacLane, Categories for the working mathematician, Graduate Texts in Math. 5, Springer-Verlag, New York, 1971.

[May96] J.P. May, Equivariant homotopy and cohomology theory, CBMS Regional Conference Series in Mathematics 91, Published for the Conference Board of the Mathematical Sciences, Washington, D.C., 1996. With contributions by M. Cole, G. Comezaña, S. Costenoble, A.D. Elmendorf, J.P.C. Greenlees, L.G. Lewis, Jr., R.J. Piacenza, G. Triantafillou, and S. Waner.

[MM02] M.A. Mandell and J.P. May, Equivariant orthogonal spectra and $S$ modules, Mem. Amer. Math. Soc. 159 (755), x+108, 2002.

[MMSS01] M.A. Mandell, J.P. May, S. Schwede, and B. Shipley, Model categories of diagram spectra, Proc. London Math. Soc. (3) 82 (2001), no. 2, $441-512$.

[Shi02] B. Shipley, An algebraic model for rational $S^{1}$-equivariant stable homotopy theory, Quart. J. Math. 53 (2002), no. 1, 87-110. 
[Shi07a] B. Shipley, $H \mathbb{Z}$-algebra spectra are differential graded algebras, Amer. J. Math. 129 (2007), no. 2, 351-379.

[Shi07b] B. Shipley, Correction to " $H \mathbb{Z}$-algebra spectra are differential graded algebras", 2007, arXiv:0708.1299v1 [math.AT].

[SS00] S. Schwede and B.E. Shipley, Algebras and modules in monoidal model categories, Proc. London Math. Soc. (3) 80 (2000), no. 2, 491-511.

[SS03a] S. Schwede and B. Shipley, Equivalences of monoidal model categories, Algebr. Geom. Topol. 3 (2003), 287-334 (electronic).

[SS03b] S. Schwede and B. Shipley, Stable model categories are categories of modules, Topology 42 (2003), no. 1, 103-153.

[Str08] N. Strickland, Is $D$ symmetric monoidal?, to appear, 2008.

David Barnes dbarnes6@uwo.ca

Department of Mathematics, The University of Western Ontario, London, Ontario N6A 5B7, Canada 